Check for updates

Cite this: J. Mater. Chem. C, 2022, 10,413

Received 14th September 2021 Accepted 9th November 2021

DOI: $10.1039 / \mathrm{d} 1 \mathrm{tc0} 4395 a$

rsc.li/materials-c

\section{Development in liquid crystal microcapsules: fabrication, optimization and applications}

\begin{abstract}
Ruixiang Qu, (D) ${ }^{a}$ Thomas F. George (D) ${ }^{b}$ and Guoqiang Li (D) *ac
A liquid crystal (LC) is an intermediate phase between a liquid and solid whose molecular orientation can be well-ordered, but the shape of the bulk LC material is like a flow. As the elastic force between the LC molecules is weak, the orientation is easily controllable by external stimuli including an electric field, light, and temperature, and thus modulates the optical properties, making them promising for various optical applications. However, the stability and versatility of LCs are severely limited by their fluidity. A series of modified LC materials such as LC polymers and LC elastomers have been designed to overcome their limitation. But the intrinsic stimuli-responsive property of LCs is severely reduced by the polymer matrix. In order to further improve the stability while maintaining the responsive property, the polymer matrix and LCs are separated to fabricate LC microcapsules (LC-Ms), which are composed of inner LC cores and outer polymer shells. This article reviews the fabrication methods, properties and applications of LC-Ms. We first discuss the emulsification and shell formation of LC-Ms, after which we introduce their intrinsic performance as well as their optimization strategies. We then illustrate three representative applications - in sensing, anti-counterfeiting, and smart fabrics. Finally, the current challenges and potential development directions of LC-Ms are introduced briefly.
\end{abstract}

\section{Introduction}

The liquid crystal, as an intermediate phase between liquids and solids, possesses liquid-like fluidity and solid-like ordering simultaneously, and has aroused wide interest due to its

\footnotetext{
${ }^{a}$ Intelligent Optical Imaging and Sensing Group, Zhejiang Lab, Hangzhou, Zhejiang 311121, China

${ }^{b}$ Departments of Chemistry \& Biochemistry and Physics \& Astronomy, University of Missouri-St. Louis, St. Louis, MO 63121, USA

${ }^{c}$ Versatile Research LLC, Columbus, Ohio 43220, USA.

E-mail: gli@zhejianglab.edu.cn
}

fascinating performance. ${ }^{1-5}$ Since 1888 when the Austrian scientist F. Reinitzer observed the first LC, more than 50 thousand kinds of LC material have been discovered. ${ }^{6,7}$ The fundamental property of the LC molecule is its anisotropic shape, which allows self-assembly and ordered arrangements of the molecules, and thus provides the material with unique anisotropy. ${ }^{8-12}$ The elastic force between LC molecules, which has been confirmed to be the main driving force of the self-assembly, is relatively weak and makes it feasible to change the orientation of the molecule. ${ }^{13-15}$ Therefore, LCs have inborn capabilities of response to external stimuli, including surface alignment, magnetic field, and electric field (above the Fréedericksz threshold) ${ }^{16-22}$ In addition, the

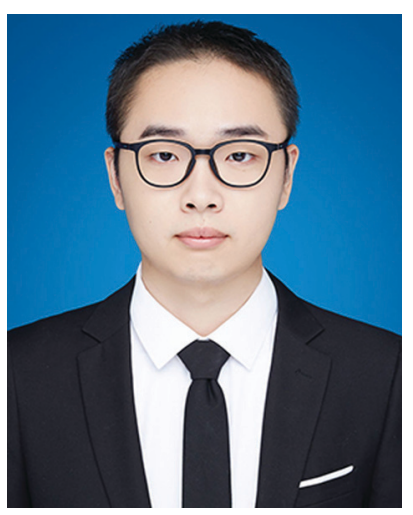

Ruixiang Qu
Ruixiang $Q u$ is currently an assistant research fellow at the Research Center for Intelligent Sensing, Zhejiang Lab. He received his BS degree in Chemistry from Tsinghua University in 2016. He then joined Prof. Lin Feng's group at Tsinghua University and received his PhD degree in 2021. His current scientific interests are focused on intelligent optical sensing based on liquid crystal materials.

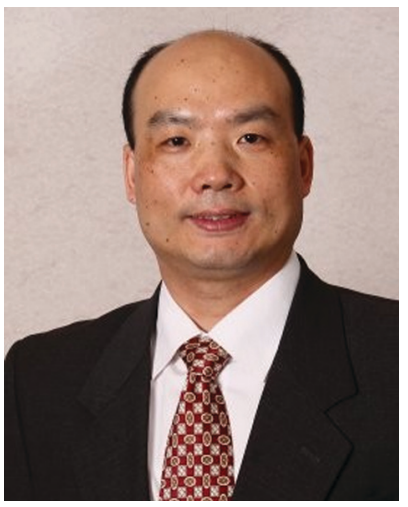

Guoqiang $\mathrm{Li}$ is currently a Professor at Zhejiang Lab. He was a faculty member and Endowed Chair at the Ohio State University, USA. His current research includes smart eyewear for vision care, liquid crystal sensors, adaptive liquid crystal optical elements, artificial retinas, and intelligent imaging of the eye with adaptive optics. He is a Fellow of Optica and SPIE. 
optical properties, such as birefringence, scattering and reflection, that are derived from the inherent arrangement of the molecules, are also basic characteristics of LCs, and can be easily observed by the naked eye using some optical techniques. ${ }^{23-28}$ Combining sensing (stimuli responsive) and expression (optical properties) together, LCs have been widely used in the fields of light modulation, displays, data storage and sensing. ${ }^{29-32}$

Despite these substantial superiorities, there remain numerous open questions for further developing LCs, among which their fluidity renders their stability and versatility. Aimed at addressing these questions, polymeric materials exhibiting liquid crystallinity have been developed, including LC polymers (LCPs), LC elastomers (LCEs), and LC networks (LCNs). ${ }^{33-36}$ Among these modified LC materials, LCPs are known as uncross-linked macromolecules that have a similar rod-like structure to traditional LCs. LCNs are densely cross-linked, showing the best mechanical properties. LCEs are the intermediate state between LCPs and LCNs, and display deformability. In comparison with traditional LCs that rely on LC cells, these modified LCs exhibit similar optical properties, while holding confined polymer matrices that can effectively impede the flow of molecules. On the other hand, although the introduction of a polymer matrix successfully decreases the fluidity, it also brings extra intermolecular forces to the LCs, such as hydrogen bonds, steric hindrance, and hydrophobic interactions, and thus reduces the stimuli-responsive properties to some extent. ${ }^{37}$ Besides, the fabrication processes of these polymeric LCs are uncontrollable, making it difficult to precisely control their microstructure, as well as studying their electro-optic performance on the microscale.

In order to overcome these drawbacks, an improved strategy, devoted to separating the polymer matrix and LCs into two phases, thus fabricating a heterogeneous material, has been proposed. A novel material known as LC microcapsules (LC-Ms) is finally obtained. The LC-Ms consist of inner LC cores and outer polymer shells, generally exhibiting a spherical geometry. ${ }^{38-40}$ The LCs in the core can remain as a fluid, conserving their sensitive responsive properties. ${ }^{41}$ The polymer shell that fully covers the core can work in one way to enhance the stability of the material, and in another way to realize the cell-free application. Another advantage of utilizing the polymer shell is to prevent the loss of dopants in the LCs, i.e., by covering dye-doped LCs (DDLCs) or metal-doped LCs (MDLCs) with the polymer shell, the dye molecules and metal ions can be confined completely, ensuring a long service life. Moreover, the LCs confined within the polymer shells can be further assembled to form various devices. For example, by filling the LC-Ms into cells, optical modulation windows can be achieved. ${ }^{42}$ By coating the LC-Ms on a fabric substrate or using the LC-Ms as a spinning material, smart wearable devices have been obtained. ${ }^{43}$ By dispersing the LC-Ms into the water phase, LC sensors with visual signals become feasible. ${ }^{44}$ Besides, each single microcapsule can be directly used as a standalone device, which further embodies the versatility of LC-Ms.

To take advantage of the LC-Ms, various encapsulation strategies have been used to fabricate LC-Ms. Several polymers suitable for capsulating LCs have been proposed and widely used. The intrinsic responsive law and optical/ electric/thermal properties, as well as the property optimization principles of LC-Ms, have been investigated in depth. In this review, we first summarize the basic knowledge about their fabrication and performance, aimed at establishing the basic concepts of LC-Ms. Some common fabrication methods, including bulk emulsification and microfluidic encapsulation, accompanied by the shell formation strategy, are exhibited in Section 2. The properties of LC-Ms, which lay the foundation for their applications, are discussed in Section 3. In addition, the relationship between the chemical composition/ microstructure and performance is illustrated to guide the precise preparation of LC-Ms. We then focus on the current hot application fields of LC-Ms, including sensors, anticounterfeiting devices and smart fabrics in Section 4. Two types of common LC-Ms, composed of nematic LCs (NLCs) and chiral nematic LCs (CLCs), respectively, are involved in the discussion simultaneously. Besides, the current challenges and potential development directions of LC-Ms are introduced in detail. The overall outline of this review is displayed in Fig. 1.

\section{Fabrication of LC-Ms}

\subsection{Fabrication of LC-in-water emulsions}

Before the encapsulation of LCs into the spherical shell, the LC-in-water emulsion must first be prepared, playing the role of the precursor. The surface energy (SE) difference between the LCs and water has been confirmed to be the key factor in the formation of emulsions. Compared with water which has a high SE $\left(\sim 71.97 \mathrm{mN} \mathrm{m}^{-1}\right.$ at $\left.25^{\circ} \mathrm{C}\right)$, LCs usually have a low SE, due to their abundant aromatic rings and aliphatic chains. As a result, surfactants added to the LC-water system tend to arrange in an orderly fashion at the surface, with their hydrophobic parts in contact with LC molecules and their hydrophilic tails in contact with the water. In this way, the early form of LC-Ms, composed of an LC yolk and a surfactant shell, was successfully achieved. At present, four different strategies - stirring, membrane emulsification, ultrasonication and microfluidic encapsulation - have been investigated in-depth to satisfy the various requirements of LC-M fabrication. ${ }^{45-47}$

Among the four strategies, stirring is the simplest and most convenient method to prepare emulsions in bulk, but it is difficult to obtain an emulsion with a narrow size distribution using this method (Fig. 2a). ${ }^{48}$ The mechanism of emulsion formation during stirring can be explained via a two-step process: (1) the continuous LCs are dispersed into tiny droplets under the shearing force; and (2) the surfactants, as the emulsifier, migrate to the LC-water interface, reduce the energy of the system, and stabilize the emulsion. Using this approach, the average emulsion size can be adjusted by the stirring rate and the stirring time, and thus affect the performance of the final LC-Ms.

Besides, ultrasonication is another frequently used method to fabricate LC-in-water emulsions (Fig. 2b). When the 


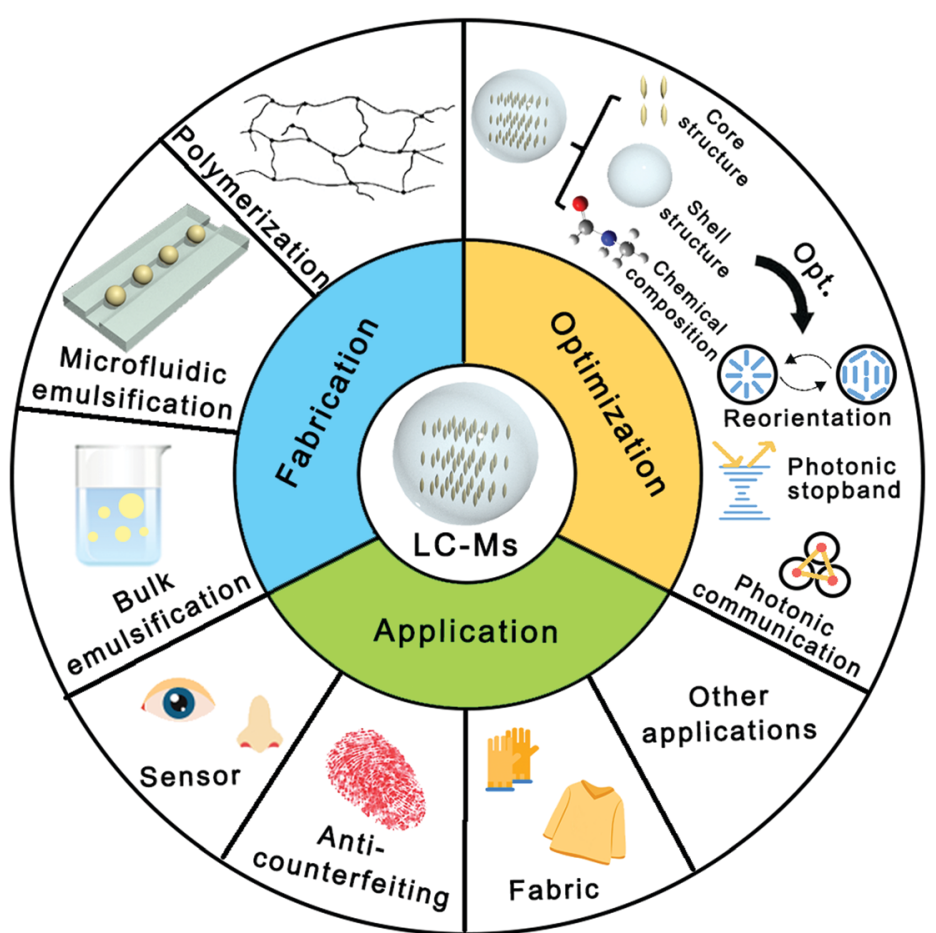

Fig. 1 Schematic showing the overall outline of this review, including the fabrication, performance optimization and application of the LC-Ms.

LC-water system is irradiated with ultrasound, cavitation, which can cause the shock wave to break the LCs into small droplets, can be observed. The minimum amplitude of the applied sound source for cavitation is called the cavitation threshold. The viscosity difference between the LCs and water is identified as the key factor for the formation of the emulsion, i.e., under the influence of ultrasonication, the phase with the lower viscosity (water) undergoes cavitation more easily and becomes the continuous phase, while the phase with the higher viscosity forms the dispersed phase. ${ }^{49-51}$ Generally, the drop size and emulsion quality of the LCs can be tuned via the irradiation time, irradiation power and position of the ultrasound source during the ultrasonication process. Nevertheless, it is also unfeasible to obtain emulsions with a uniform size via ultrasonication.

In order to achieve a narrow size distribution, membrane emulsification has emerged as a promising technique for the production of a dispersed phase that has a uniform size ranging from hundreds of nanometers to tens of micrometers (Fig. 2c) ${ }^{52}$ During membrane emulsification, the membrane with a consistent pore size is used as the emulsion template. The LCs are pushed out through the template from one side and emulsified into the water on the other side. The droplet size of the LCs fabricated via membrane emulsification is directly related to the pore size of the template.

With the development of LC-Ms, there is an increasing requirement for monodispersed LC droplets. Therefore, microfluidics has been utilized to obtain droplets with a precisely controlled size and composition. ${ }^{53-55}$ In the 1950s, Skegges et al. accomplished an analytical chemistry experiment in a microchannel; since then studies using microfluidics have developed steadily. ${ }^{56}$ In 1998 , the soft lithography of polydimethylsiloxane provided a reliable method for constructing microchannels. ${ }^{57,58}$ At the present time, there are two different microchannels prepared via soft lithography, including crossjunction geometry and axis-symmetric geometry, which have great advantages for the preparation of LC-in-water emulsions (Fig. 2d-g).

The cross-junction geometry drives the formation of the LC droplet via the following steps. First, water flows through the main channel as the continuous phase, and the LCs enter by injection through the lateral channel as the dispersed phase. Then, the LC-water interface is formed at the cross-junction, with the LCs being distorted into droplets by the pressure gradient. Finally, the LC-in-water emulsion is assembled in the channel. ${ }^{59}$ In order to improve the preparation efficiency of the cross-junction geometry, a series of cross-junctions can be integrated together to construct a parallel line.

In addition, the axis-symmetric geometry consists of two or more coaxially assembled glass capillaries. The simplest axissymmetric geometry is the co-flow geometry in which the LCs flow inside the inner capillary and the water flows through the outer capillary in the same direction. ${ }^{60}$ Individual monodisperse LC droplets can be formed at the tip of the inner capillary. The tip-to-tip geometry is an improved geometry for preparing emulsions. In contrast to co-flow capillary devices, an extra capillary, which is spatially symmetric with the original inner capillary, is introduced to the system, thus providing a flowfocusing effect to facilitate the generation of stable drops. ${ }^{61}$

It is worth mentioning that, using microfluidics, other emulsions with more complex structures, such as doubleemulsions and triple-emulsions, have been successfully achieved. 


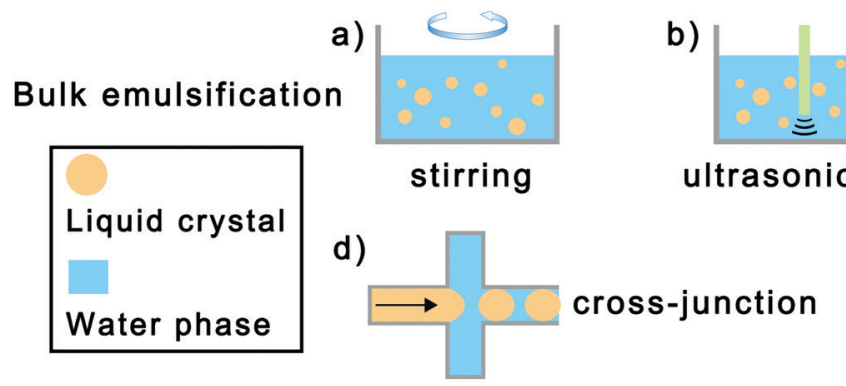

Microfluidic emulsification

f)

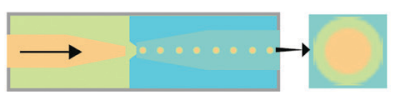

g)

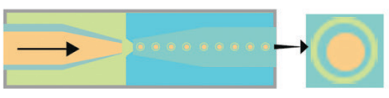

tip-to-tip double emulsion tip-to-tip triple emulsion

h)

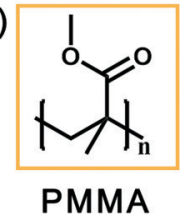

i)

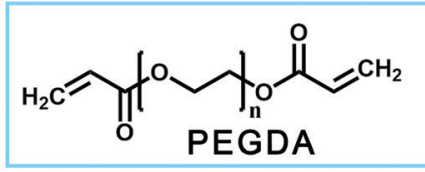

c)

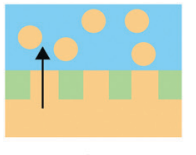

membrane

e)

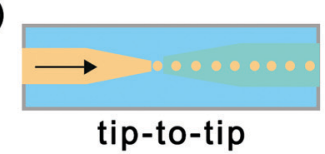

Shell composition

Common hydrogels
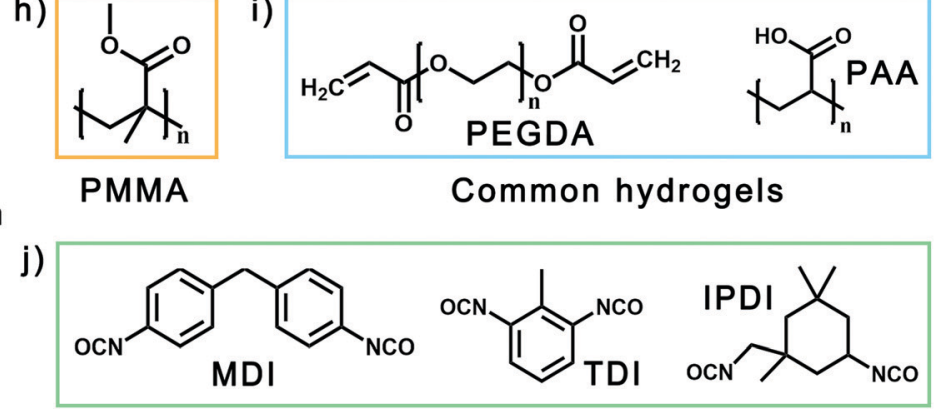

Common PU monomers

Fig. 2 Schematics showing the fabrication process of liquid crystal microcapsules: (a) stirring emulsification, (b) ultrasonication emulsification and (c) membrane emulsification are the bulk emulsification strategies; (d) microfluidic emulsification using a cross-junction microchannel; (e)-(g) microfluidic emulsification using a tip-to-tip microchannel, fabricating single emulsion, double emulsion and triple emulsion, respectively; (h) polymethyl methacrylate (PMMA), (i) hydrogels and (j) polyurethane (PU) monomers are the common composition of the polymer shell.

In a typical single LC-in-water emulsion, the monomers leading to the formation of the polymer shell are mixed in the LC phase, which will reside in the LCs and have a negative impact on the optical properties. In comparison, in the complex emulsion, the LCs and monomers are localized in the dispersed phase and the middle phase, respectively. Thus, the optical-property deterioration brought on by the residual monomers is effectively avoided. For example, using microfluidics, a triple-emulsion that consists of an innermost CLC core, a water alignment layer, a photocurable oil layer, and a continuous water phase has been realized by Lee et al. in which the elastic polymer shell is assembled via photopolymerization of the photocurable oil layer, and polyvinyl alcohol (PVA) is dissolved in the water layer to promote the planar alignment. ${ }^{62}$ The fabrication device contains three coaxial capillaries nested in a square capillary. The obtained CLC-Ms exhibit a satisfactory photonic cross-communication pattern, indicating their well-aligned orientation. Besides, by constructing a LC-in-water-in-LC-in-water emulsion, it is feasible to integrate two different CLCs with opposite handedness together, which further confirms the versatility of the microfluidic strategy. ${ }^{63}$

Although the advantages of microfluidics have been discussed emphatically, their existing problems should not be ignored, among which the most urgent one is the low production capacity, which restricts the practical application of microfluidics. In addition, pretreatment of the capillary also increases the workload of the microfluidic strategy. As a result, a new emulsion fabrication strategy that has comprehensive advantages should be further developed to fabricate LC-Ms that have high size uniformity, stability, and optical performance with high throughput.

\subsection{Shell formation}

After the LC-in-water emulsions are fabricated, polymerization reactions at the LC-water interface are required to obtain the cured shell, which is vital to the stability of the LC-Ms Considering the requirements in the reaction region (at the LCwater interface) and the shell transparency, alternative polymer candidates are actually limited. Up to now, there have been three kinds of polymer shell that are widely used - polymethyl methacrylate (PMMA), hydrogel and polyurethane (PU) - each of which exhibits unique monomers and structure. ${ }^{64-67}$ Some of the simple LC-Ms, so-called LC droplets, that exhibit fluid shells derived directly from surfactants, such as SDS and CTAB, have also been investigated in depth. ${ }^{68,69}$

PMMA, known as organic glass, has the advantages of high transparency and low cost, and is promising in the field of chemical mediators, building materials and adhesives (Fig. 2h). A common method for fabricating PMMA-capsulated LC-Ms is solvent-evaporation-induced phase separation (SIPS). ${ }^{70}$ In the 
SIPS process, PMMA is co-dissolved with the LC in an organic solvent, which plays the role of the dispersed phase in the LC-in-water emulsion. Then, the organic solvent is removed by evaporation at a specific temperature, and the PMMA spontaneously migrates to the LC-water interface to form a solid shell. Free-radical polymerization that is triggered by ammonium persulfate, with methyl methacrylate as the monomer, is also a candidate for fabricating the PMMA shell.

Hydrogels are a class of network polymer derived from the cross-linking reaction and show diverse compositions and interactions with water (Fig. 2i). A typical hydrogel can swell rapidly in the water phase and control the diffusion of water. Such a characteristic has been utilized to ensure the long-term stability of LC-Ms. For example, after the UV light-induced photopolymerization of poly(ethylene glycol) diacrylate (PEGDA), LC-Ms with the PEGDA hydrogel membrane have been successfully fabricated. ${ }^{71}$ The water molecules in the hydrogel membrane drain slowly, leading to infiltration of the oil phase into the core through the water-depleted membrane, thereby dissolving the LCs. In order to solve this problem, the as-prepared LC-Ms are dispersed in water to ensure that the hydrogel is fully swollen, thus preventing penetration of the LC. In addition, the acacia-gelatin hydrogel fabricated via coacervation-induced crosslinking has been confirmed as an ideal candidate for the shell. ${ }^{72}$

The PU shell, fabricated via the copolymerization of PU isocyanate and polyhydric alcohols, shows a flexible performance, which varies according to the monomers. A variety of isocyanate monomers have been utilized to suitably fabricate the PU shell, including diphenyl-methane-diisocyanate (MDI), toluene diisocyanate (TDI) and isophorone diisocyanate (IPDI) (Fig. 2j). ${ }^{73-75}$ PVA, a polyhydric alcohol that facilitates planar alignment of the LC molecules, can also participate in the polymerization reaction. Driven by the PVA, an orderly orientation can be achieved simultaneously during the polymerization process, which guarantees a surfactant-free strategy, thereby simplifying the preparation process. Such a strategy has been utilized to construct a temperature-responsive color display. ${ }^{76}$ However, the transparency of the PU shell is relatively low, which calls for further development of PU-containing LC-Ms and for further modification of the molecular structure.

\section{Properties of LC-Ms}

\subsection{Stimuli-responsive configuration transition}

Owing to the unique orientation sensitivity possessed by LCs, LC-Ms possess satisfactory stimuli-responsive properties. External stimuli such as interfacial molecules, electric and magnetic fields, and light can possibly disrupt the preferred orientation of the LC cores. Different from the free LCs that are immobilized by the LC cells, which tend to exhibit homeotropic orientation, planar orientation and disordered orientation, the LCs encapsulated in the spherical shell are re-orientated under the influence of the shell, and thus can produce the radial configuration, the bipolar configuration, or the pre-radial configuration. ${ }^{77}$ Common surfactants such as CTAB, DTAB, and SDS favor the radial configuration of the LC cores, because the hydrophobic interaction between the surfactants and the LC molecules can trigger the directional arrangement of the surfactants at the core-shell interface, with their hydrophilic head combined with water, and their hydrophobic tail combined with the LC. ${ }^{78}$ Therefore, the LC molecules tend to be perpendicular to the polymer shell. By contrast, some ordered polymers, such as PVA and polyimide (PI), can lead to the bipolar configuration due to tangential surface anchoring. ${ }^{79}$ Besides, molecules at the interface can change the orientation of the LC cores by combining with the orientation agents. For example, biomolecules, including nucleic acids and proteins, as well as heavy metal ions, possess electrostatic interactions with ionic surfactants and can disrupt the configuration of the LCs.

The electric field-induced LC orientation relies on the electric field force applied to the molecules. When the LC molecules are exposed to an electric field, electrodes are generated in the molecules, and the positive centers in the molecules tend to move to the negative charge while the negative centers in the molecules tend to move to the positive charge. The dipole moment, which is a measure of the distance between the positive/negative centers, mainly decides the magnitude of the force. ${ }^{80}$ Such an electric field-induced reorientation phenomenon has been widely used for smart windows/fabrics based on LCs and LC-Ms. ${ }^{81,82}$ Moreover, the magnetic field-induced LC orientation, which is related to the magnetic moment of the LCs, affects the configuration of the LC cores via a similar mechanism. For chiral nematic liquid crystal microcapsules (CLC-Ms), the magnetic field or electric field can interact with the helical superstructure, rendering the change of the helix, and influence the color of the microcapsules. ${ }^{83}$

In some of the photosensitive group-modified LC-Ms, the configuration of the LC core can be controlled via light illumination. Azo dyes, which have $\pi$-conjugated systems, are a series of synthetic dyes that contain azo groups. Most of the azo dyes possess an obvious $\pi-\pi^{*}$ transition under UV irradiation, which can lead to breaking and rotation of the azo bond. ${ }^{84}$ As a result, azo dyes can realize cis-trans isomerism under light irradiation. By doping the azo dyes into the LC core, the orientation of the LCs can be adjusted during the isomerization process. ${ }^{85,86}$ The driving force of the orientation is the dipole interaction between the dye molecules and the LC molecules. Moreover, by introducing the azo group into the LC molecules in situ, the photoinduced orientation can be achieved without the doping of the dyes, which is in favor of the transmittance of the LC-Ms. Besides, by employing a chiral dopant with the azobenzene group, preparing CLC-Ms with light-responsive properties is feasible. ${ }^{87}$ The cis-trans isomerism of the azo bond can cause a change in the helical twisting power (HTP), and thus tune the pitch of the CLC core. By utilizing the above configuration transition strategies properly, the application of LC-Ms in the field of anti-counterfeiting, sensing, photonic inks and other intelligent devices will be feasible. 


\subsection{Photonic stopband and photonic communication}

CLCs have been confirmed to be an ideal photonic crystal as they exhibit a photonic bandgap (PBG) along the axis, which can selectively reflect light, and color the CLCs when the stopband is in the visible range. ${ }^{88,89}$ Being composed of photonic crystals and transparent polymer shells, CLC-Ms also possess a photonic stopband and show diverse colors under either the naked eye or polarized optical microscopy. The central wavelength of the reflected light depends on the incident angle and the intrinsic properties of the CLC core. This can be estimated using Bragg's law,

$$
\lambda=n P \cos \theta
$$

where $n$ is the average refractive index of the LC, $P$ is the pitch of the CLC, and $\theta$ is the incident angle. ${ }^{90}$ At normal incidence, the bandwidth depends on the refractive index and the pitch of the photonic crystal, and can be calculated according to the following:

$$
V \lambda=P\left(n_{\mathrm{e}}-n_{\mathrm{o}}\right)
$$

Here, $n_{\mathrm{o}}$ and $n_{\mathrm{e}}$ are the ordinary and extraordinary refractive indices of the LCs. ${ }^{41}$ The easiest way to tune the photonic stopband of CLC-Ms is to regulate the amount of chiral reagent, which decides the pitch directly.

The photonic stopband can also be tuned via the temperature, magnetic field and electric field. Considering that the polymer shell effectively improves the thermal stability of the CLC, the temperature-induced photonic stopband variation has been investigated thoroughly to fabricate thermochromic CLC-Ms. ${ }^{91,92}$ Increasing the temperature can, on the one hand, increase the pitch by promoting the phase transition, and, on the other hand, decrease the pitch by improving the solubility of the chiral reagent, therefore exhibiting an integrated impact on the photonic stopband. It is worth mentioning that the photonic stopband also shows selectivity to the handedness of the incident circularly polarized light, i.e., the left-handed CLC core can only reflect left circularly polarized light, while right circularly polarized light is reflected by the right-handed CLC core. ${ }^{93}$ The high selectivity of the photonic stopband endows the CLC-Ms with potential in the field of LC displays and anti-counterfeiting.

When the CLC-Ms are arranged in a close-packed way, the reflected light from one microcapsule can act as the incident light for neighboring microcapsules, and subsequently is reflected again according to Bragg's law, causing the photonic cross-communication phenomenon. ${ }^{94}$ In the photonic crosscommunication system, the CLC-Ms show bright central dots and additional radial lines simultaneously under polarized optical microscopy. The central dots are derived from the normal reflection, and the radial lines are derived from the double reflection. Interestingly, the cross-communication pattern is closely related to the arrangement, size and pitch of the microcapsules, and therefore can act as an optical fingerprint. In a photonic cross-communication system containing thousands of CLC-Ms, each tiny change in the spatial position or the geometrical shape will lead to a variation of the pattern. Such an optical fingerprint is thought to establish the relationship between LC-Ms and information storage, specific identification and data transmission.

\subsection{Performance optimization}

The performance of LC-Ms can be easily tuned by adjusting their chemical composition and microstructure. Here, considering that the two variables impact the performance at the same time, we will simplify the discussion by controlling the variables. When the chemical composition of the LC-Ms is fixed, the microstructure has a great impact on the threshold electric field, transmittance, response time and phase-transition temperature; by contrast, the chemical composition of the LC-Ms is closely related to the interaction between the shell and the core and also influences the aforementioned performance when the microstructure is fixed. In the following paragraphs, we will discuss these relationships in depth.

The microstructure of the LC-Ms can be described by the core size and shell thickness, which have been quantified in the form of equations in the reported studies. The core size of the LC-Ms is calculated using the equation

$$
r=\left(\frac{3}{4 \pi} \frac{W_{\mathrm{LC}}}{\rho_{\mathrm{LC}}}\right)^{\frac{1}{3}}
$$

where $W_{\text {LC }}$ and $\rho_{\text {LC }}$ are the weight and density of the LC core, respectively. The shell thickness $(t)$ of the LC-Ms is calculated via

$$
t=\left[1-\left(\frac{C_{\mathrm{LC}} / \rho_{\mathrm{LC}}}{\left(1-C_{\mathrm{LC}}\right) / \rho_{\text {shell }}+C_{\mathrm{LC}} / \rho_{\mathrm{LC}}}\right)^{\frac{1}{3}}\right] R
$$

where $C_{\mathrm{LC}}$ is the weight percentage of the LC core, and $\rho_{\mathrm{LC}}$ and $\rho_{\text {shell }}$ are the density of the LC core and the polymer shell, respectively. ${ }^{95}$ It is worth mentioning that the LC cores and LC-Ms are simplified into a normal sphere in the above two equations.

The threshold electric field $\left(E_{\mathrm{th}}\right)$, which is used to measure the responsive sensitivity, depends heavily on the anchoring elastic forces between the LC molecules, and thus is related to the core size. Considering an isolated spherical LC core within a polymeric shell, and assuming that the LCs are isotropic, the relationship between the $E_{\text {th }}$ and the core size can be estimated as:

$$
E_{\text {th }}=\frac{1}{3 r}\left[\frac{\delta_{\mathrm{LC}}}{\delta_{\text {shell }}}+2\right]\left[\frac{K\left(l^{2}-1\right)}{\varepsilon_{0} \Delta \varepsilon}\right]^{\frac{1}{2}} .
$$

In this equation, $r$ is the core size of the LC-Ms, and $\delta, \varepsilon, K$ and $l$ are the constants that describe the conductivity, dielectric anisotropy, elastic force and shape anisotropy, respectively. ${ }^{96,97}$ Obviously, $E_{\text {th }}$ shows a negative correlation with the core size. Sheng et al. fabricated a series of LC-Ms with different core sizes and shell thicknesses and compared the practical $E_{\text {th }}$ values with the theoretical calculations. ${ }^{98}$ It was found that the practical $E_{\text {th }}$ value is higher than the theoretical value. 
Such a phenomenon is ascribed to the interface anchoring effect brought on by the polymer shell, which mainly hinders the rotation of the LC molecule in the shell material edge, resulting in a higher $E_{\text {th }}$ value. Apart from the interface anchoring effect, the polymer shell can also increase the $E_{\text {th }}$ value via the shielding effect. Under the shielding of the polymer shell, the electric field that is actually applied to the LC core is smaller than the external electric field. ${ }^{99,100}$ The thicker the polymer shell is, the greater the shielding effect will be. As a result, increasing the core size and decreasing the shell thickness will be of benefit for obtaining LC-Ms with a low $E_{\text {th }}$. However, increasing the core-shell ratio will in the meantime reduce the stability of the LC-Ms, and thus lead to breaking of the microcapsules. Such a trade-off between stability and sensitivity calls for the subtle regulation on the microstructure.

Moreover, the shielding effect and the anchoring effect of the polymer shell can also affect the phase-transition temperature. In essence, the phase-transition of the LCs is the process of weakening the elastic force and increasing the molecular mobility. The anchoring effect of the shell can impede the molecular movement at the core-shell interface, and thus increase the phase-transition temperature. ${ }^{101}$ Furthermore, a longer response time, longer delay time, lower transmittance and lower responsive yield will be observed with an increase of the shell thickness. And the influence of the core size on the performance is opposite to the shell thickness.

Besides, when the microstructure of the LC-Ms is fixed, the chemical composition, which decides the surface free energy, can also affect the responsive process of the microcapsules. ${ }^{102}$ The surface free energy of the shell can be calculated according to the equation:

$$
\gamma=\gamma^{\mathrm{p}}+\gamma^{\mathrm{d}}
$$

where $\gamma^{\mathrm{p}}$ and $\gamma^{\mathrm{d}}$ are the polar and non-polar parts of the surface free energy. When the polymer shell has a large $\gamma^{\mathrm{p}}$, it can show a natural electric dipole moment even without the applied electric field under the influence of the asymmetric positive/negative charge center. Therefore, a dipole-dipole interaction between the polar polymer shell and the LC is formed. Thus, a high driving voltage, high phase-transition temperature, and long response time are necessary to break the interaction.

The reversion process of the $\mathrm{LC}$ is dependent on the adhesion power between the polymer shell and the LC core, which can be described as:

$$
W_{\mathrm{ab}}=2\left(\sqrt{\gamma_{\mathrm{a}}^{\mathrm{d}} \gamma_{\mathrm{b}}^{\mathrm{d}}}+\sqrt{\gamma_{\mathrm{a}}^{\mathrm{p}} \gamma_{\mathrm{b}}^{\mathrm{p}}}\right) .
$$

In this equation, $W_{\mathrm{ab}}$ is the adhesion power, and $\gamma_{\mathrm{a}}$ and $\gamma_{\mathrm{b}}$ are the surface free energy of the LC core and the polymer shell, respectively. ${ }^{103}$ When the core and shell have a similar polarity, the adhesion power will be strong, and thus lead to a long delay time. As a result, to obtain LC-Ms with a short delay time, the combination of a low-polarity core/high-polarity shell or a low-polarity shell/high-polarity core can be ideal candidates. If we further take the driving voltage and phase-transition temperature into account, the low-polarity shell/high-polarity core appears to be the optimal option.

\section{Applications of LC-Ms}

\subsection{Sensors}

Owing to the sensitive stimuli-responsive properties of the LCs, their application in the field of sensing has been widely explored, for the detection of specific information and its transformation into the required form, so as to meet the requirements of information-transmission, -processing, -storage, -display, -recording and -control. ${ }^{104-106}$ Traditional sensors cannot express the signal directly, making it highly dependent on the output system. ${ }^{107,108}$ By contrast, the LC sensor is a superior device that integrates the detection and expression together, and thus can greatly simplify the instrument. However, the fluidity of the LC molecules severely limits the ease of processing and structural stability, restricting their applications and durability. Overcoming these limitations by replacing free LC molecules with LC-Ms is a crucial approach. The sensing properties of a typical LC sensor depend on three key factors: ${ }^{109}$ first, the orientation of the LCs is sensitive to the external stimulus, i.e., the preferred orientation can be disrupted by either an asymmetric force or steric hindrance from the environment. Second, the unique optical properties of LCs, especially their birefringence and Bragg reflection, can effectively modulate the signal, which can be expressed visually. Last but not least, the molecular arrangement of thermotropic LCs can change as the temperature changes, and a phase change occurs at a specific temperature. Fortunately, it has been found that encapsulating LCs into spherical shells will not affect the above characteristics, and therefore many scientists are focusing on the study of LC-M sensors.

Up to now, according to the fluidity difference of the shell, two different kinds of LC-M sensors have been proposed. One uses LC-Ms with a cured shell, and the other one, so-called LC droplets, exhibits a fluid shell with high fluidity, among which the former sensor shows better stability and versatility, while the latter sensor can be easily produced. Although there is an obvious difference between them, the two kinds of sensors hold similar advantages. Apart from the stability mentioned above, these sensors show a large surface area-to-volume ratio, which benefits their detection sensitivity and improves the detection limit. By subtly designing the structure and the composition of the LC-M sensors, the detection of temperature, proteins, nucleic acids, heavy metal ions, bile acids, glucose and other common molecules has been successfully achieved. ${ }^{110,111}$ Shown in Table 1 is a summary of the state-of-the-art studies on LC-M sensors.

Hong et al. developed a LC-Ms sensor via a simple emulsification-volatilization process. $^{112}$ The cationic surfactant, trimethyl octadecyl ammonium bromide, is utilized to impact the anchoring of the LCs at the LC-aqueous interface. Using a $\mathrm{Hg}^{2+}$-binding aptamer, the LC-M sensor shows mercury ion 
Table 1 Summarization of liquid crystal microcapsule-based sensors

\begin{tabular}{|c|c|c|c|c|c|}
\hline Core & Shell type & Shell composition & Detection object & Sensing principle & Ref. \\
\hline $5 \mathrm{CB}$ & Fluid shell & $\begin{array}{l}\text { Anti-IgG anchored poly(styrene- } b \text { - } \\
\text { acrylic acid) }\end{array}$ & IgG antigen & $\begin{array}{l}\text { The anchoring of IgG changes the orientation } \\
\text { of liquid crystal }\end{array}$ & 110 \\
\hline $5 \mathrm{CB}$ & Fluid shell & $\begin{array}{l}\text { Tetradecyltrimethylammonium } \\
\text { bromide }\end{array}$ & $\mathrm{Hg}^{2+}$ & $\begin{array}{l}\text { The specific binding of } \mathrm{Hg}^{2+} \text { and aptamer } \\
\text { lead to the release of surfactant }\end{array}$ & 112 \\
\hline $5 \mathrm{CB}$ & Fluid shell & $\begin{array}{l}\text { Tetradecyltrimethylammonium } \\
\text { bromide }\end{array}$ & $\mathrm{Pb}^{2+}$ & $\begin{array}{l}\text { The specific binding of } \mathrm{Pb}^{2+} \text { and DNAzyme } \\
\text { lead to the release of surfactant }\end{array}$ & 113 \\
\hline $5 \mathrm{CB}$ & Fluid shell & $\begin{array}{l}\text { Poly(vinyl alcohol)/sodium dodecyl } \\
\text { sulfate }\end{array}$ & Bile acids & $\begin{array}{l}\text { The continuously flowing bile acids can } \\
\text { remove SDS from the surface of 5CB droplets, } \\
\text { leading to the instability of 5CB droplets. }\end{array}$ & 114 \\
\hline $\begin{array}{l}\text { CLC mixture of ZLI- } \\
2293 \text { and MLC- } 6248\end{array}$ & Fluid shell & CLC & $\mathrm{H}_{2} \mathrm{O}_{2}$ & $\begin{array}{l}\mathrm{H}_{2} \mathrm{O}_{2} \text { oxidizes luminol in the presence of } \\
\text { catalytic metal ions, resulting in the } \\
\text { chemiluminescence }\end{array}$ & 118 \\
\hline $\begin{array}{l}\text { CLC composed of } \\
\text { MLC-2132 and CB15 }\end{array}$ & Fluid shell & $\begin{array}{l}\text { Poly(vinyl alcohol) } \\
\text { Sodium dodecyl sulfate } \\
\text { Poly(acrylic acid) }\end{array}$ & $\begin{array}{l}\mathrm{pH} \\
\text { Glucose } \\
\text { Cholesterol }\end{array}$ & $\begin{array}{l}\text { Enzymes oxidize glucose/cholesterol into } \mathrm{H}^{+} \text {, } \\
\text { and liquid crystal change the configuration via } \\
\text { protonation and deprotonation of the PAA }\end{array}$ & 120 \\
\hline
\end{tabular}

$\left(\mathrm{Hg}^{2+}\right)$ detection properties in an aqueous medium (Fig. 3a). The $\mathrm{Hg}^{2+}$-binding aptamer can form a hairpin structure with $\mathrm{Hg}^{2+}$, weakening the interaction between the surfactant and the aptamer molecules, resulting in the self-assembly of the surfactant as well as the homeotropic orientation of the LCs. By contrast, the organization of the surfactant molecules is disrupted by the electrostatic interactions between the positively charged surfactant and the negatively charged a)
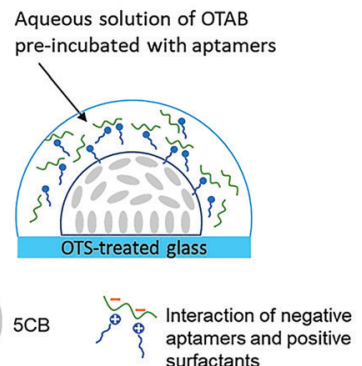
surfactants

c)

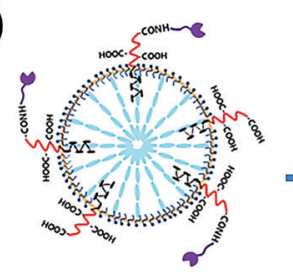

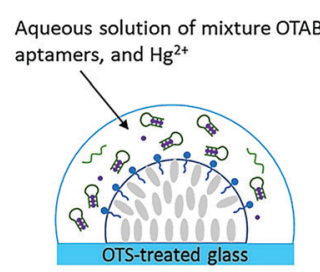
Hairpin structure
formation by the $\mathrm{T}_{-} \mathrm{Hg}^{2+}{ }_{-} \cdot \mathrm{Hg}^{2+}$
$\mathrm{T}$ complex

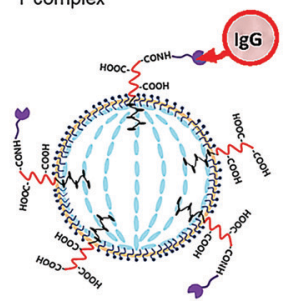

b)
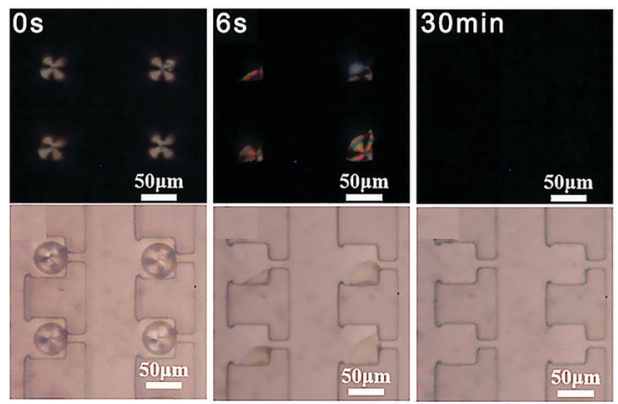

d)

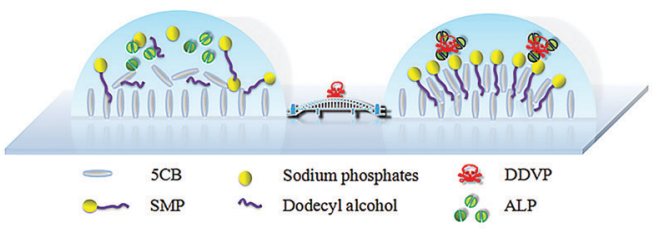

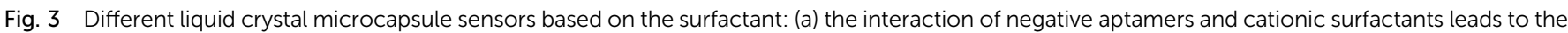

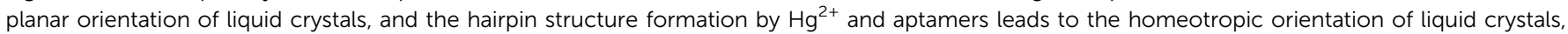

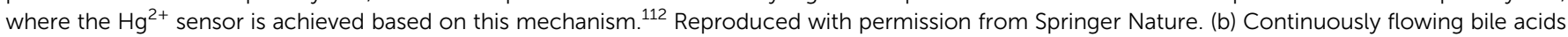

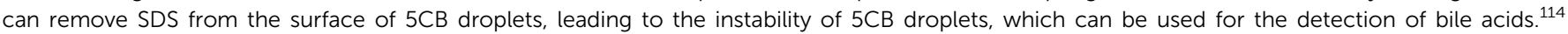

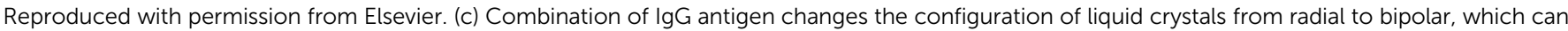

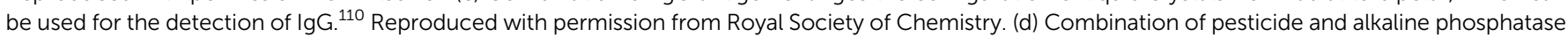
leads to the reorientation of liquid crystals, which can be used for fabricating pesticide sensors. ${ }^{116}$ Reproduced with permission from Elsevier. 
aptamer in the absence of $\mathrm{Hg}^{2+}$. The detection results can be easily observed under a polarized optical microscope, as the optical responses of either a bright fan-shaped pattern or a dark-crossed pattern. Such a strategy shows satisfactory sensitivity (a low detection limit of $100 \mathrm{pM}$ ) and outstanding selectivity, indicating its potential in real-time and convenient $\mathrm{Hg}^{2+}$ detection in the aqueous phase. A similar strategy is utilized to realize the detection of $\mathrm{Pb}^{2+} \cdot{ }^{113} \mathrm{~A}$ single-stranded DNAzyme, which can bind with $\mathrm{Pb}^{2+}$ specifically, is chosen as the aptamer. The resultant sensor is also sensitive and specific.

Apart from the cationic surfactant, SDS, as a typical anionic surfactant, is also a possible candidate for fabricating LC-M sensors. Recently, a novel microfluidic device composed of monodisperse 4-cyano-4'-pentylbiphenyl (5CB) and PVA/SDS stabilizers has been developed for the on-chip detection of bile acids (Fig. 3b). ${ }^{114}$ In the digestive system, bile acids act as an emulsifier, which can remove lipids from the fatty food interface and promote the formation of micelles, accelerating the uptake of fatty food by intestinal mucosal cells. ${ }^{115}$ As a result, in such a LC-surfactant system, the flowing of bile acids can remove the sodium dodecyl sulfate shell from the LC-Ms, and thus cause collapse of the microcapsules.

The detection result can be observed using a polarized optical microscope. Furthermore, by subtly selecting the binding aptamer, such a LC-surfactant-aptamer system can also realize the detection of proteins, DNA, and other metal ions. For example, the optical response of stearic-acid-doped 5CB microcapsules is utilized for the real-time and label-free detection of heavy metal ions, where anti-IgG-anchored LC-Ms have been realized to detect IgG, and a 5CB droplet platform based on the alkaline phosphatase hydrolysis of sodium monododecyl phosphate has been established to achieve the sensitive identification of organophosphate pesticide (Fig. 3c and d). ${ }^{110,111,116}$ The director configurations of LCs in those LC-Ms sensors are essentially largely dictated by the surface anchoring and the elastic energy between the LC molecules.
As a consequence, the detection properties can be easily optimized by adjusting the size of the LC-Ms, the concentration of the anchored surfactant, and the structure of the surfactant/ LC, making the system extendable and flexible.

Whereas the aforementioned devices are cladded by fluid shells, another type of LC-M sensor, with cured shells, also shows extraordinary sensing ability. Owing to the thermostability brought about by the cured shells, these sensors have shown aptitude for the detection of temperature. As shown in Fig. 4a, Lee et al. encapsulated CLCs with a robust hydrogel membrane via a microfluidic approach, and explored their application in thermosensitive photonic inks. ${ }^{71}$ The hydrogel shells of the microcapsules are fabricated via photoinitiated polymerization involving PEGDA. The as-prepared microcapsules show thermochromic properties, displayed as a green color at room temperature and exhibiting a red-shift as the temperature is increased. In this way, the temperature change can be discernible even with the naked eye. Further study has confirmed that the reflection color of the CLC-Ms is tunable in situ by simultaneously injecting two distinct CLC solutions to form a single core, which can provide a large-sized color-library. It is worth mentioning that the freestanding CLC-M sensor can form a suspension in an aqueous phase, so that the local temperature of the aqueous phase can be measured separately.

It should be noted that the color-change mechanism of photonic crystalline microcapsules is complex. There are two factors that directly influence the pitch of the CLC and further determine the color of the microcapsules. On the one hand, with an increase in temperature, the solubility of the chiral agent in the CLC increases, which tends to reduce the pitch and the wavelength of the reflected light. On the other hand, the CLC tends to relax and unwind with increasing temperature, which instead increases the pitch, leading to a red-shift of the reflected light. As a result, different photonic crystalline microcapsules usually show different color-change principles. For example, Lin et al. have developed a photonic crystalline
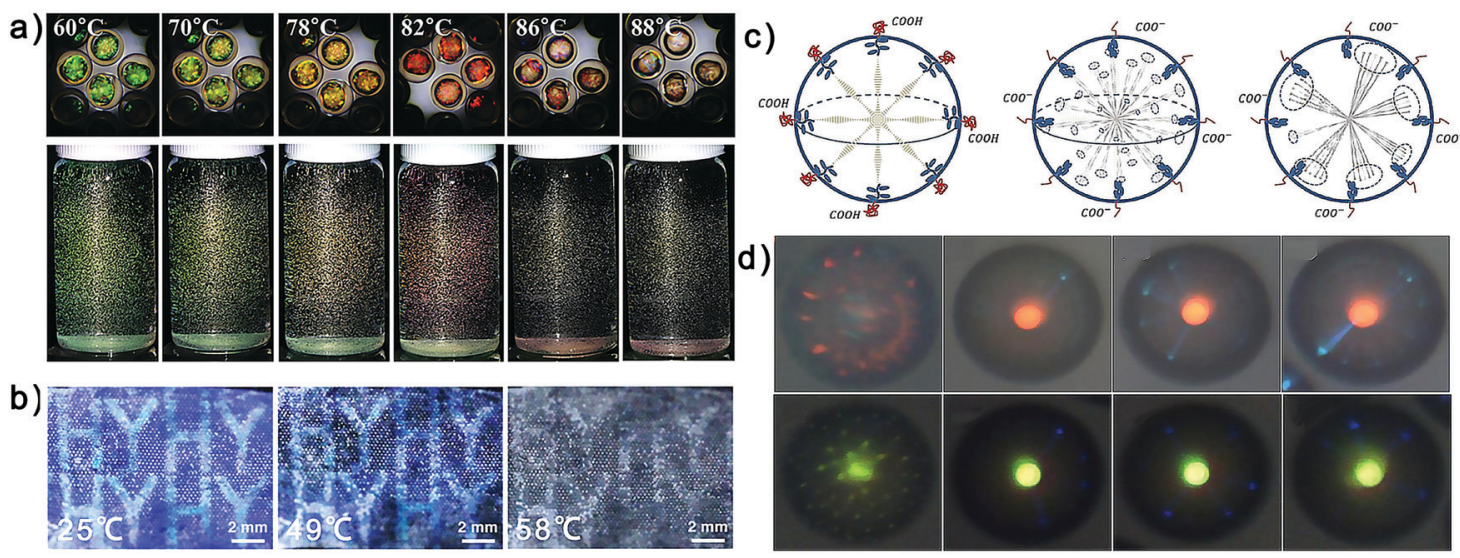

Fig. 4 Different liquid crystal microcapsule sensors containing chiral nematic liquid crystals: (a) the temperature sensor derived from liquid crystal microcapsules showing thermochromic properties. ${ }^{71}$ Reproduced with permission from Wiley. (b) Expressing temperature signals in the form of a picture. ${ }^{76}$ Reproduced with permission from Elsevier. (c) Liquid crystal microcapsule $\mathrm{pH}$ sensor showing different configurations at different $\mathrm{pH}$ values; and (d) in the presence of enzymes, the liquid crystal microcapsule $\mathrm{pH}$ sensor can be used for glucose and cholesterol detection. ${ }^{120}$ Reproduced with permission from the American Chemical Society. 
microcapsule by encapsuling different CLCs into PU shells, which are derived from the interfacial polymerization of TDI and tetraethylenepentamine (TEPA). ${ }^{117}$ The LC-Ms show a fixed color when the temperature is lower than the phase-transition temperature, but become transparent and colorless directly without any wavelength-shift phenomenon when the temperature rises above the phase-transition temperature.

With the development of LC-M temperature-sensor technology, temperature-responsive color displays that can show different pictures under different temperatures have been proposed (Fig. 4b) ${ }^{76}$ After encapsulating the CLCs into PU shells, different RGB microcapsules are transferred into a PU elastomer stencil with an array of designated holes, which is prepared via photolithography in combination with the etching technique, using a colloidal packing-driven microcapsule registry. Each kind of microcapsule is arranged at a specific site to form a particular pattern, and exhibits satisfactory temperature-responsive colorchange properties. In this way, the temperature signal is expressed vividly in the form of words or pictures.

Apart from the surfactant-based molecular detection and pitch-based temperature detection mentioned above, some chemical reactions with high specificity have also been utilized to construct LC-M sensors. To be more specific, the chemical reactions involved in the sensing can be divided into two categories, where one can generate light or heat during reactions, and directly express macroscopic signals, while the other tends to indirectly express signals via the subsequent reactions. For instance, the luminol reaction has been used for hydrogen peroxide detection. ${ }^{118}$ Luminol (5-amino-2,3-dihydro1,4-phthalazinedione) is a chemiluminescent reagent that can be oxidized by an oxidizing agent, after which a cyclic peroxide can be generated, and can further react to generate 3 -aminophthalic acid. When the 3 -aminophthalic acid is transformed from the excited state to the ground state, blue fluorescence can be observed. ${ }^{119}$ Based on this mechanism, Iwai et al., constructed CLC core-shell microcapsules consisting of a CLC material as the middle phase, an aqueous luminol solution as the inner phase, and an aqueous PVA solution as the outer phase via a microfluidic strategy. It has been confirmed that when the photonic band gap edge is near the emission peak, the luminescent enhancement is most effective. Thus, the photonic band gap of the CLC is subtly adjusted to raise the chemiluminescence emission maximum and enhance the sensitivity of the sensor. Although this work has preliminarily realized the combination of the luminol reaction and a LC sensor, the fluorescence effect is still too weak for the quantitative and stable measurement of oxidizing agents. Some countermeasures, such as fabricating CLC-Ms with both right- and left-handed CLC materials and introducing dyes with a fast and narrow emission, are needed to improve such a system. It is foreseeable that with further development of the luminol strategy, an alternative approach for the detection of metal ions $\left(\mathrm{Cu}^{2+}, \mathrm{Fe}^{2+}, \mathrm{Mn}^{2+}\right.$ etc., as catalysts of the luminol reaction) and hemoglobins (which contain $\mathrm{Fe}^{2+}$ ) will become available.

The aforementioned luminol reaction can directly express detection signals. Some other reactions, which indirectly express signals via the subsequent reactions, have also been investigated in depth. A pH-sensor has been developed by coating CLC with poly(acrylic acid)-b-poly(4-cyanobiphenyl-4'oxyundecyl acrylate) (PAA- $b$-LCP). ${ }^{120}$ As displayed in Fig. $4 c$ and d, the protonation/deprotonation of PAA is the key factor for realizing $\mathrm{pH}$ detection. When the $\mathrm{pH}$ value is high, the deprotonated PAA is rich in negative charge, leading to the swelling of the PAA, which favors the homeotropic orientation of the CLC. By contrast, when the $\mathrm{pH}$ value is low, the protonated PAA is in a neutral charge state, leading to shrinking of the PAA, which favors the radial orientation. The change in the orientation can be observed under a polarized optical microscope as either a bright central spot or randomly distributed small spots. By combining the above $\mathrm{pH}$-sensor and some typical enzymatic reactions, indirectly expressed detection of biomolecules becomes feasible. An enzymatic reaction, such as the "lock and key" reaction which is dominated by efficient biochemical catalysts, holds the advantage of being sensitive, specific and fast, and has been the most reliable approach for identifying biomolecules. ${ }^{121}$ Glucose oxidase and cholesterol oxidase are immobilized on the PAA shell of the above $\mathrm{pH}$-sensor via an EDC/NHS reaction. When the modified sensor meets glucose or cholesterol, the enzymatic reaction occurs, which generates $\mathrm{H}^{+}$to protonate the PAA shell, leading to the reorientation of the CLC. Such a strategy can also be utilized for the detection of other molecules. For example, by immobilizing the reactants of enzymatic reactions on the PAA shell. By blocking the enzymatic reaction with heavy metal ions, the detection of heavy metal ions can be realizable. Some other enzyme inhibitors can also be detected using the same approach.

The detection of gases, such as volatile organic compounds and inorganic gaseous substances, shows important theoretical and practical values in the field of environmental protection and monitoring. ${ }^{122}$ The interaction between $\mathrm{Cu}^{2+}$ and different gases has been widely used for gas sensing. Niu $e t$ al. modified $\mathrm{Cu}^{2+}$ on a glass substrate via cross-linking between glutaraldehyde and chitosan, followed by assembling the glass into a LC sensor. ${ }^{123}$ Complexation of the nitrile groups (-CN) of 5CB with $\mathrm{Cu}^{2+}$ favors the homeotropic orientation of the LC. When the sensor detects ammonia, a competitive reaction between ammonia and $5 \mathrm{CB}$ occurs, leading to the binding of ammonia to the $\mathrm{Cu}^{2+}$ and changing the alignment of the LC. Besides, Sridharamurthy et al. realized the detection of dimethyl methylphosphonate (DMMP) using a similar mechanism. ${ }^{124}$ Upon exposure to DMMP vapor, the DMMP diffuses through the film of the LCs and binds to $\mathrm{Cu}\left(\mathrm{ClO}_{4}\right)_{2}$, resulting in the rearrangement of the LC. It is worth mentioning that these studies mainly focus on traditional cell-based LCs with limited durability and sensitivity, whereas few efforts have been devoted to the fabrication of LC-M gas sensors. There are two reasons for the hindered development of such materials. First, the immobilization of $\mathrm{Cu}^{2+}$ on the shell of the microcapsules remains unsolved. Designing and fabricating a transparent cross-linked polymer that can chelate $\mathrm{Cu}^{2+}$ seems to be a potential solution. Besides, the selectivity of such a strategy is relatively low, as the combination of 
$\mathrm{Cu}^{2+}$ and gas shows low specificity. Once these problems have been solved, LC-M gas sensors will probably show extraordinary capabilities in trace gas detection. Apart from the $\mathrm{Cu}^{2+}$-gas hybrid, some other interactions are also promising in the design of LC-M gas sensors, such as the specific recognition between the $\mathrm{CO}_{2}$ receptor ribulose-1,5-disphosphate and $\mathrm{CO}_{2}$, the reaction between dinitrogenase and $\mathrm{N}_{2}$, and the chromogenic reaction undergone by formaldehyde. ${ }^{125-127}$

\subsection{Anti-counterfeiting}

Different from traditional labels, which are designed mainly to make goods attractive, anti-counterfeiting labels are supposed to help people reduce economic loss that is threatened by counterfeiting. The next-generation anti-counterfeiting technologies are established in security inks that are able to offer various optical states to express identifiable information. ${ }^{128,129}$ Owing to their unique optical properties, in particular the polarization-dependent reflection enabled by the photonic stopband, CLC-Ms have been used for anti-counterfeiting inks in recent years. Besides, a new type of LC-M, the so-called fluorescent CLC microcapsule (FCLC-M), which is composed of CLC-Ms and fluorescent materials, is also suitable for anti-counterfeiting because of its ability to express distinct but intact information in reflective and fluorescent states. A summary of the LC-Ms used for anti-counterfeiting activities is shown in Table 2.

In 2013, Guo et al. fabricated a CLC-M film with a doublehanded circularly polarized light reflection band via interfacial polymerization (Fig. 5a and b). ${ }^{130}$ In order to obtain the CLC films with a double-handedness structure, CLC-Ms with rightand left-handed structures are mixed together and coated on the glass substrate. The morphology of the CLC-Ms is subtly adjusted by optimizing the core/shell ratio, emulsification rate, agitation rate and emulsifier content. The as-prepared CLC-M film displays temperature-dependent light-reflection properties. When the temperature is $25{ }^{\circ} \mathrm{C}$, two different reflection bands can be observed due to the difference in the pitch lengths of the CLCs with left- and right-handed structures. However, when the temperature is $45^{\circ} \mathrm{C}$, both L-CPL and R-CPL are reflected within the same reflection band, which is caused by a change in pitch length. Seo et al. fabricated a solid-state CLC-M to make anticounterfeiting patches via the photopolymerization of reactive mesogens (RMs) in CLC droplets. ${ }^{131}$ The curing of RMs provides the CLC-Ms with a 3D rigid network, which leads to a satisfactory mechanical stability. Based on the stable CLC-Ms, an anticounterfeiting patch was prepared using two distinct CLC-Ms with the same reflection colors but opposite handedness, which displays different letters under different circularly polarized light sources.

In order to increase the confidence level, fluorescent materials have been doped into CLC-Ms for the in situ expression of the fluorescent signal and the RGB signal. DC5, a cyano-substituted oligo( $p$-phenylene vinylene) derivative, which is free of aggregation-induced quenching effect and emits intense light with a high fluorescence quantum yield in both the solution and solid states, is regarded as an ideal doping candidate. ${ }^{132}$ The resultant fluorescent CLC microcapsules (FCLC-Ms) display two colors through distinctly different mechanisms: the structural color is caused by the pitch length, while the fluorescent color is caused by the molecular structure of DC5 (Fig. 5c). A geminate "security label", which exhibits the colorful "Christmas tree" in reflective mode under white light and shows the cyan QR code in fluorescent mode, has been created based on the FCLC-Ms by filling the pixel array using different microcapsules, thus providing a versatile platform for the encryption and protection of valuable authentic information. Besides, a similar FCLC-Ms has been fabricated by doping rare-earth complexes (RECs) into CLCs, showing three types of reversible responsive behavior: including thermotropic reflection evolution, temperaturedependent fluorescence emission and Fredericks electro-optical response. ${ }^{133}$ The stability of the FCLC-Ms is greatly improved by the $\pi-\pi$ interaction between the aromatic rings on the RECs and CLC, which endows the material with a promising application potential. The as-prepared FCLC-Ms provide a triple-protection strategy because they integrate three different state-of-the-art photonic elements together.

In addition, the photonic cross-communication property of CLC-Ms has also been utilized to realize anti-counterfeiting, showing the advantages of being easy-to-read and inimitable. ${ }^{134}$ Due to their unique optical characteristics and intrinsic randomness, the photonic cross-communication between CLC-Ms is regarded as the ideal candidate for realizing a physical unclonable function (PUF). Geng et al. fabricated microcapsules with a CLC shell via microfluidic technology followed by a UV-curing process. ${ }^{135}$ It is emphasized that the optical quality of the traditional CLC droplets remains unsatisfactory, which severely hinders their practical applications.

Table 2 Liquid crystal microcapsules used for anti-counterfeiting

\begin{tabular}{|c|c|c|c|c|}
\hline Core & Shell type & Shell composition & Anti-counterfeiting principle & Ref. \\
\hline $\begin{array}{l}\text { CLCs doped with rare-earth } \\
\text { complexes }\end{array}$ & Polymer shell & $\begin{array}{l}\text { Polyurethane shell pre- } \\
\text { pared from TDI and TEPA }\end{array}$ & $\begin{array}{l}\text { The CLC integrates reflection mode, fluorescent } \\
\text { mode and electro-optical response together }\end{array}$ & 133 \\
\hline $\begin{array}{l}\text { CLC mixture with } \\
\text { left-handed and right- } \\
\text { handed helical structure }\end{array}$ & Polymer shell & $\begin{array}{l}\text { Polyurethane shell } \\
\text { prepared from isophorone } \\
\text { diisocyanate }\end{array}$ & $\begin{array}{l}\text { The CLCs reflect both L-CPL and R-CPL while their } \\
\text { wavelength trends are opposite }\end{array}$ & 130 \\
\hline $\begin{array}{l}\text { CLC composed of E7 and } \\
\text { chiral dopants }\end{array}$ & CLC shell & $\begin{array}{l}\text { CLC cross-linked by } \\
\text { reactive mesogen }\end{array}$ & $\begin{array}{l}\text { The patch shows different letters under different } \\
\text { circularly polarized light sources }\end{array}$ & 131 \\
\hline DC5-doped CLC & Fluid shell & PVA & $\begin{array}{l}\text { The label shows different patterns under reflective } \\
\text { and fluorescent modes }\end{array}$ & 132 \\
\hline Water & CLC shell & Cured CLC & $\begin{array}{l}\text { The photonic cross-communication between CLC- } \\
\text { Ms forms a physical unclonable function (PUF) }\end{array}$ & 135 \\
\hline
\end{tabular}




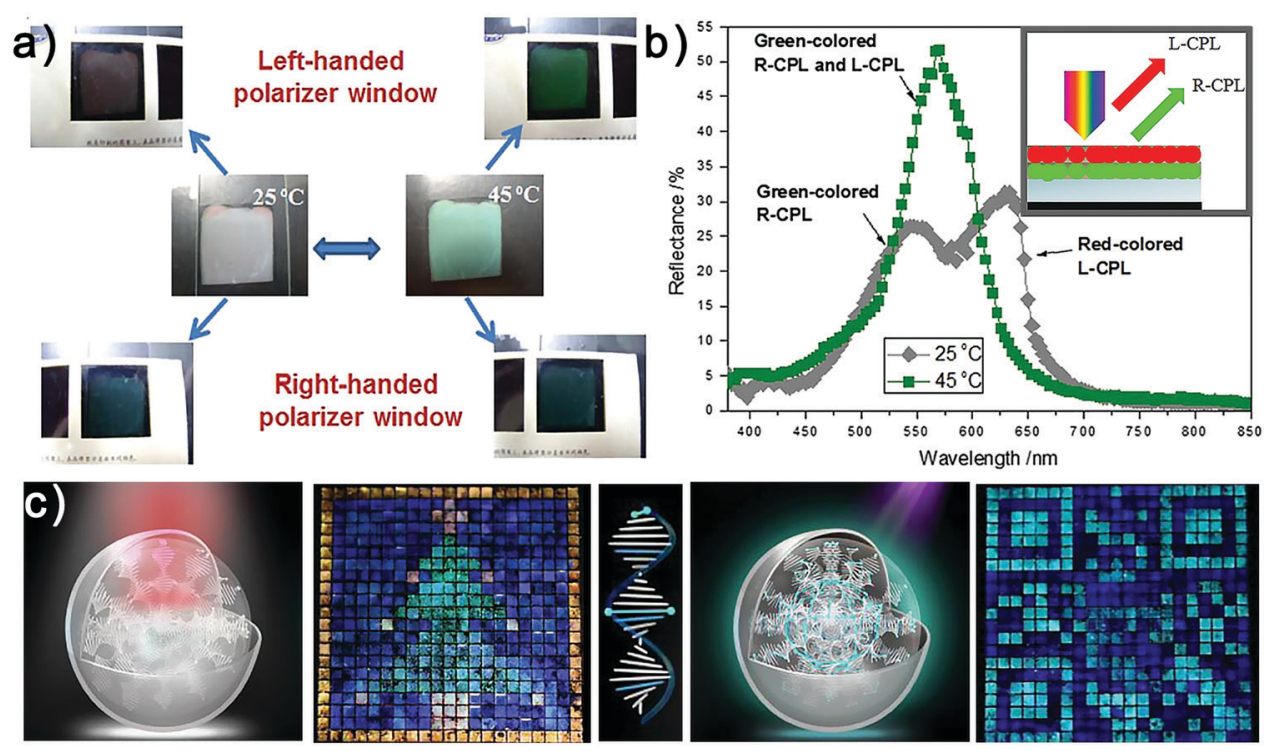

Fig. 5 Liquid crystal microcapsules used for anti-counterfeiting: (a) and (b) the anti-counterfeiting film, made from two different kinds of chiral nematic liquid crystal microcapsules, shows different colors under different circularly polarized light sources and different temperatures. ${ }^{130}$ Reproduced with permission from Royal Society of Chemistry. (c) Liquid crystal microcapsules doped with fluorescent molecules show different patterns under different light sources. ${ }^{132}$ Reproduced with permission from Springer Nature.

However, a transition from droplet to shell can ensure a sharp pattern as well as excellent optical quality, and thus solves the problem mentioned above. Moreover, by combining CLC shells of various pitch, thickness and chirality, enormous variation possibilities are available to the system, which further enhances the security level. Such a PUF derived from photonic cross-communication is believed to constitute an interesting component for realizing future high-security authentication and identification protocols.

\subsection{Textiles and fibers}

Wearable devices as portable devices that can be directly worn or integrated into clothes can realize abundant functions through software support and data interaction, and have led to important changes in our daily life. ${ }^{136-138}$ Since Google Glass was released in 2012, a large number of wearable devices have come out one after another, encompassing many fields such as healthcare, entertainment and education. Possessing satisfactory electro-stimulated responsive properties and thermochromic properties, LCs are supposed to become the foundation of newgeneration wearable devices, which exhibit the characteristics of being low cost, simple to preparate and visually readable. However, traditional LCs show high fluidity and celldependence, making them unsuitable for processes such as spinning and spraying, which are the common processes for preparing wearable textiles and fibers. ${ }^{139-142}$ The development of LC-Ms seems to be a partial solution, especially considering that the stability of LC-Ms make them suitable for spinning/ spraying, either by being mixed with fibrous scaffolds such as polyvinyl pyrrolidone (PVP) and polyethylene oxide (PEO), or by direct use. Shown in Table 3 is a summary of the textiles and fibers derived from LC-Ms. Although there have been a variety of studies focused on LC-M textiles and fibers in recent years, there remain some crucial problems that impede the development of this field. For example, the optical quality of LCs suffers from an inevitable decrease after the LC is processed into a textile. Besides, the driving voltage of the LCs will increase significantly due to the shielding effect of fibrous scaffolds. Moreover, the washability

Table 3 Textiles and fibers derived from liquid crystal microcapsules

\begin{tabular}{|c|c|c|c|c|c|}
\hline Core & Shell type & Shell composition & Processing method & Function & Ref. \\
\hline Dye-doped LC with $\mathrm{Fe}_{3} \mathrm{O}_{4} @ \mathrm{SiO}_{2}$ & Polymer shell & Polymethyl methacrylate & Electrospin & $\begin{array}{l}\text { Electro-responsive fabric with color } \\
\text { enhancement effect and magnetically } \\
\text { controllable properties }\end{array}$ & 145 \\
\hline E7 doped with diverse dyes & Polymer shell & Polymethyl methacrylate & Wet spinning & $\begin{array}{l}\text { Fibers with color change properties } \\
\text { under an electric field }\end{array}$ & 146 \\
\hline Dye-doped E7 & Polymer shell & Polymethyl methacrylate & Coating & $\begin{array}{l}\text { Textile showing color change } \\
\text { properties under an electric field }\end{array}$ & 144 \\
\hline $5 \mathrm{CB}$ & Polymer shell & Polyvinylpyrrolidone & $\begin{array}{l}\text { Printing and multi- } \\
\text { spinning }\end{array}$ & $\begin{array}{l}\text { Record and image of human hand } \\
\text { temperature }\end{array}$ & 153 \\
\hline Dye-doped LC and paraffin & Polymer shell & Polymethyl methacrylate & Wet spinning & Bistable electrochromic & 147 \\
\hline
\end{tabular}


and antifouling properties of the LC-M textile still need to be promoted.

Compared with other LC-Ms materials, wearable textiles usually have higher requirements for color purity. At the present stage, two different strategies have been employed to meet these requirements. One is to dope the dichroic dye, which can absorb light as the electric field of light is along the long axis of the dye molecules, into the LCs to form a guesthost mixture in which the permanent dipole moment of the LC mesogens will work on the dichroic dye molecule to generate an induced dipole moment. As a result, the alignment of the dichroic dye molecules will change with the change of the LC alignment, making it possible to tune the color of the textile via external stimuli such as a magnetic field, electrical field and light. ${ }^{143}$ The other strategy is to utilize thermochromic CLCs and provide the textile with thermochromic properties. Combining the textile and sensor technologies together, a new type of device, a so-called wearable sensor, is also developed, and preliminary applications have been made in body-temperature detection and disease prevention.
As shown in Fig. 6a-c, Sheng et al. have fabricated electrochromic dye-doped LC microcapsules (DDLC-Ms) via an emulsion polymerization strategy, and have further processed them into a DDLC-based smart fabric through a simple printing strategy. ${ }^{144}$ When the current is switched off, molecules in the DDLC-Ms are randomly oriented with the light absorbed and scattered by the dichroic dye molecules. As a result, the smart fabric shows a red color under direct view and via a microscope. By contrast, when the current is switched on, the red color disappears and the fabric become turbid. Considering that the threshold voltage and the driving voltage of the DDLC-Ms can be adjusted by altering the content of the DDLCs inside the microcapsules, a considerable effort has been made to adjust the fabrication conditions of the DDLC-Ms, especially the type of emulsifier which affects the surface morphology, particle size, and size distribution, as well as the stirring rates and emulsification periods. Finally, dispersant-0402 and DNS-86 are found to be conducive to achieving uniform particle size distributions, and a stirring rate of $1000 \mathrm{rpm}$ for $60 \mathrm{~min}$ is regarded as the optimal emulsification conditions, which leads to the DDLC-Ms with a

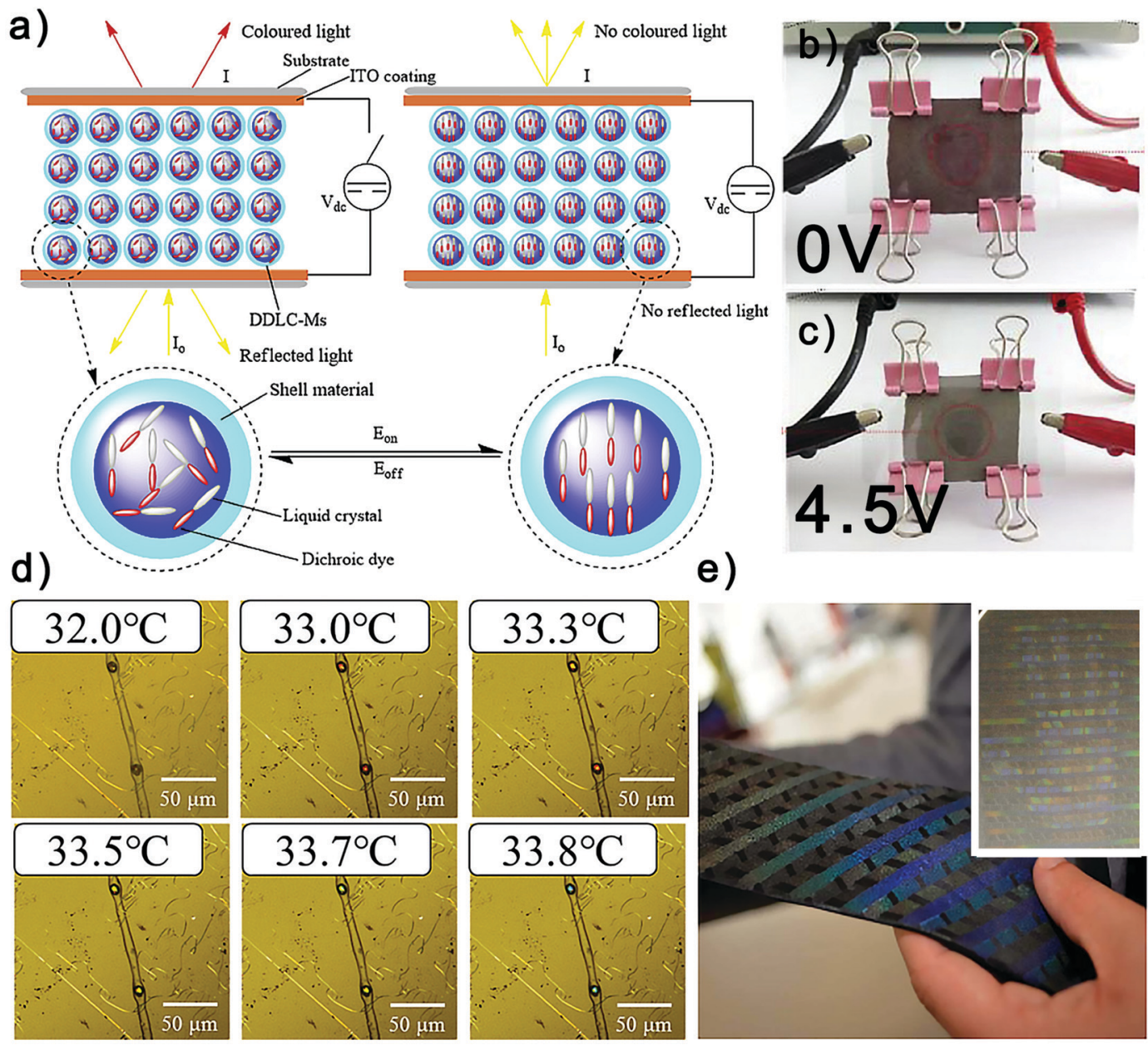

Fig. 6 Functional textiles and fibers made from liquid crystal microcapsules: (a)-(c) dye-doped liquid crystal microcapsules can absorb light in the absence of an electric field and can be used for fabricating electrochromic fabrics. ${ }^{144}$ Reproduced with permission from Elsevier. (d) Thermochromic fiber derived from liquid crystal microcapsules. ${ }^{72}$ Reproduced with permission from Elsevier. (e) Thermochromic liquid crystal tie, which can display the image of a human hand, has been achieved using liquid crystal microcapsules. ${ }^{153}$ Reproduced with permission from Wiley. 
$68.58 \mathrm{wt} \%$ core loading, an average size of $3 \mu \mathrm{m}$ and a $3.6 \mathrm{~V}$ driving voltage.

Although the aforementioned DDLC-Ms has been confirmed to be an effective candidate to protect DDLCs, there remain some tough problems including the limited solar radiation modulation range and the high near-infrared radiation transmittance. In order to solve these problems, $\mathrm{Fe}_{3} \mathrm{O}_{4}$ @$\mathrm{SiO}_{2} @ V T E S @ D D L C @ P M M A$ with a novel yolk-shell structure is reported. ${ }^{145}$ The utilization of $\mathrm{Fe}_{3} \mathrm{O}_{4}$ particles, as the yolk, on the one hand provides the material with multispectral absorption properties, and on the other hand makes it possible to remotely control the microcapsules using a magnetic field. However, the driving voltage and maximum transmittance are severely impacted by the semiconductor yolk, which needs to be further improved. Besides, inspired by plant respiration, a seed emulsion polymerization strategy has been crafted to realize multicolor DDLC yolk-shell microcapsules. By subtly adjusting the reaction temperature, diverse dichroic dyes displaying different colors are successively encapsulated into the PMMA shell and are isolated in separate layers by P(S-EDGMA-TFEMA). ${ }^{146}$ Further study confirmed that the outer dichroic dye has an electric field shielding effect on the internal one, leading to different driving voltages, even if both use the same LC. Based on the original microcapsules, a functional fiber is fabricated via wet spinning. The fiber can switch from bluish violet to purple, red, and colorless states by controlling the DC current. Recently, inspired by the morphology-switch of tardigrades, a novel DDLC/paraffin-Ms, prepared via a one-step emulsion solvent diffusion/evaporation method with the PMMA shell, has been proposed to achieve a controllable DDLC orientation anchor effect, which can realize the bistable electrochromic function of DDLC displays. ${ }^{147}$ Paraffin plays the role of the phase-change material in the microcapsules, which can greatly increase the spatial hindrance at room temperature, and thus ensure the stability of the isotropic and anisotropic states of the LCs. The microcapsules show the reversible color change from gray-red to colorless with a low driving voltage $(\sim 7.4 \mathrm{~V})$. And both states can remain stable for more than one week after removing the electric field. Such a material is promising in the field of bistable LC displays, smart fabrics, and electroresponsive sensors.

Apart from the DDLC-Ms with photoelectric properties, another type of textile, which is composed of CLC and shows thermochromic properties, has also been studied in depth. Although a large number of studies have been devoted to directly processing CLCs, it has been found that the CLC cannot be uniformly distributed in the textile, which seriously decreases the thermochromic performance. ${ }^{148-150}$ As a consequence, CLC-Ms have been designed to solve the above problem. Guan et al. encapsulated CLCs in an acacia-gelatin coacervate shell, and fabricated the CLC-Ms with a responsive temperature at about $35{ }^{\circ} \mathrm{C}$ (Fig. 6d). ${ }^{72}$ The pitch of the LCs is controlled at $300 \mathrm{~nm}$, because the optimum reflection from a CLC requires about 8 twists. After adding the CLC-Ms to the PVP solution and processing them into CLC-Ms/PVP fibers, the thermochromic performance remains satisfactory. Besides,
Hao et al. mixed two kinds of CLCs including cholesteryl oleyl carbonate (COC) and cholesterol pelargonate (CPE) in a specific proportion, and fabricated CLC microcapsules with a responsive range between $33.4{ }^{\circ} \mathrm{C}$ and $38.0{ }^{\circ} \mathrm{C}$, which is compatible with human comfort. ${ }^{151}$ Then, the CLC-Ms are coated on the woven cotton fabric using a coating binder. In addition, the treated fabric shows the same responsive temperature. This work confirms the feasibility of adjusting the thermochromic properties of the textile simply by mixing CLCs with different phase-transition temperatures together. Although the above studies have realized the fabrication of thermochromic textiles, it was found that their luminosity and color purity decreased a lot after the CLCs had been processed, which is indeed an imperative for improvement.

A special type of LC-Ms, so-called liquid-crystal-clad fiber, has also been developed as a wearable textile. ${ }^{152}$ The fiber is fabricated by cladding conventional monofilament fibers with low-molecular-weight CLCs stabilized by an outer polymer sheath. The monofilament fibers in this material can, on the one hand, provide mechanical strength, and on the other hand adsorb the transmitted light, thereby enhancing the contrast of the colored fibers. Owing to their columnar geometry, the reflected wavelength of the fibers shows a dependence on the viewing angle, which can be explained using Bragg's Law. Finally, the fibers are woven into a complex fabric that can respond to temperature.

The intensive study in LC-M-functionalized textiles has promoted the development of the wearable sensors, which exhibit an application potential in body-temperature detection and disease prevention. The most severe challenge in preparing a wearable sensor lies in the trade-off between a high sensitivity and a wide response range. West et al. printed different patterns of multiple thermochromic LC-Ms that have different and adjacent responsive temperatures on a single fabric and preliminarily broke the trade-off dilemma. ${ }^{153}$ The patterns provide a visual temperature reference that allow high sensitivity and a broad range, and realized the recording and imaging of a human hand temperature. Besides, the LC-Ms can also be sprayed or printed on a tie as a fashion improvement (Fig. 6e). For disease prevention, a variety of LC-based wearable sensors have also been proposed, including a bracelet consisting of a CLC and a black bandage, which can detect the arteries and veins in the wrist, as well as a smart sock made from CLC fabrics for diabetes monitoring. ${ }^{43}$ In order to further promote the development of such devices, the durability of the textile and the microcapsules is significant. The introduction of covalent bonds between the fabric substrate and the microcapsules, as well as between the shell and core of the microcapsules, appears to be a feasible strategy.

\subsection{Other applications}

The various chemical compositions and adjustable microstructures endow LC-Ms with versatile characteristics. As a result, they have shown exceptional performance in many other fields such as biomimetic materials, micropumps, drug delivery and biolabeling, which further demonstrate the potential applications of 
LC-Ms. A micropump derived from the LC-Ms was developed by Fleischmann et al. in 2012. ${ }^{154}$ Integrating two state-of-the-art LC technologies together - CE and LC-Ms - the subtly designed material can realize the reversible pumping out and absorption of a liquid core by actuation of the LCE shell in a jellyfish-like motion (Fig. 7a and b). A capillary tip is utilized to pierce the LCE shell to help the pumping process. When the temperature is higher than the isotropic transition temperature, the punctured elastomer changes its shape and reduces the core volume, leading to the expulsion of the liquid core. By contrast, upon cooling the material, the LCE reverts back to its original shape, and the liquid is sucked back to the core. Such an elastomer can be integrated into a microfluidic system as a micropump, which can greatly simplify the components of the system. Furthermore, it is believed that by further modifying the elastomer, pumping of the liquid can be controlled using other stimuli. For example, by incorporating azo bonds in the LCE, the process can be triggered by light irradiation; in addition, by dispersing magnetic nanoparticles in the LCE, electromagnetic irradiation offers an extra path to induce actuation.

Besides, Spillmann et al. fabricated a LC-Ms delivery system via a thermal polymerization approach, realizing drug delivery and bio-labeling simultaneously. ${ }^{155}$ A LC cross-linker and a perylene-based dye are utilized to fabricate the LC core, which endow the material with fluorescence. And the outer shell is comprised of a carboxyl-terminated polymerizable surfactant. The LC-Ms are further covalently coupled to the iron transport protein transferrin via NHS-EDC reaction to facilitate their endocytic uptake by human embryonic kidneys. Owning to the hydrophobic nature of the LC core, the LC-Ms can act as a host for doxorubicin, and thus realize the carrying and intracellular release of doxorubicin, which can be tracked via the fluorescence signal. It is worth mentioning that the fluorescence emission spectrum of the LC-Ms can be tuned by changing the mole percentage of the dye relative to the $\mathrm{LC}$, laying the foundation for performing multi-color labeling. Such a delivery system displays the possibility of utilizing LC-Ms as a satisfactory medium for simultaneous cellular imaging, tracking and drug delivery. Recently, a water-in-oil-in-water double emulsion with a photo-responsive NLC shell was realized by Iwai et al., which shows the release action of the inner droplet triggered by UV light irradiation, and also has potential in controlled drug-release systems. ${ }^{156}$

Bionics, born at the interface of biological and materials sciences, has been a feasible and widely used approach to develop functional LC materials. Yang et al. reported the fabrication of 3D-printed biomimetic systems exhibiting synergetic color- and shape-responsive properties by dispersing CLC droplets in a PVA matrix (Fig. 7c and d). ${ }^{157}$ Upon heating the systems, the CLC droplets change into an isotropic state, leading to simultaneous variations in color and shape. The color change is attributed to the temperature-sensitive helical pitch of the CLC; the shape change is dominated by the variable geometrical shape of the droplets. With an increase in temperature, the oblate droplets tend to become spherical, resulting in volume contraction along the matrix plane and volume dilation perpendicular to the matrix plane. Based on this mechanism, a biomimetic octopus, which can adapt its color and shape to match that of the surrounding environment, as well as a biomimetic flower, which can blossom under certain temperatures, have been discussed.

Blue phase liquid crystals (BPLCs) are ordered materials with double twist cylinder structures, which exist over a very narrow temperature range. The response time of BPLCs is usually in the submillisecond region, making them promising for many electro-optical applications that require rapid
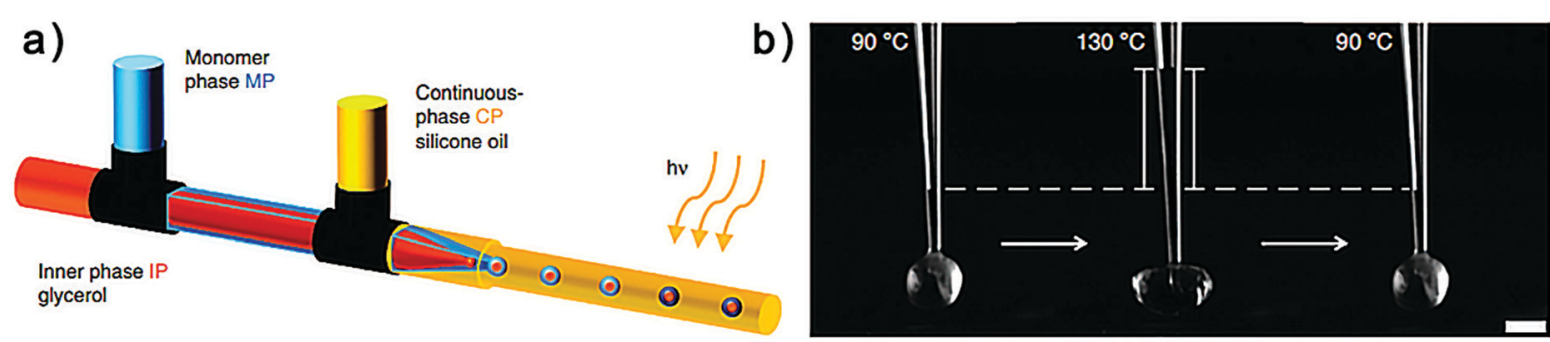

c)

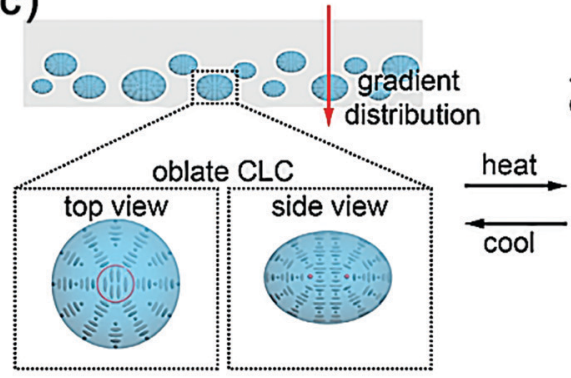

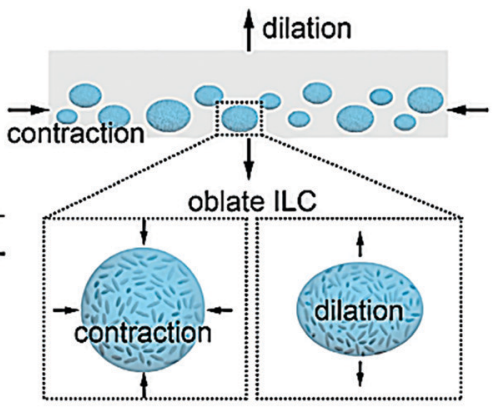

d)
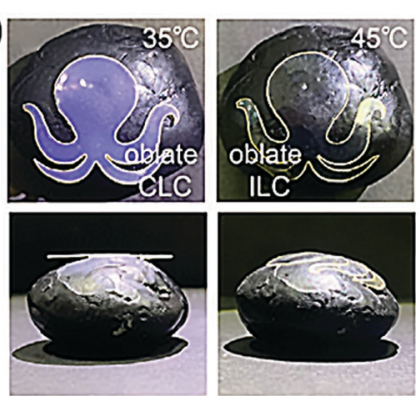

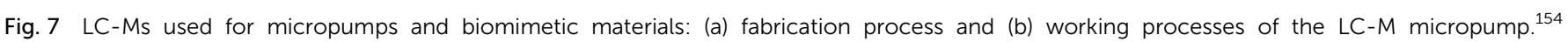

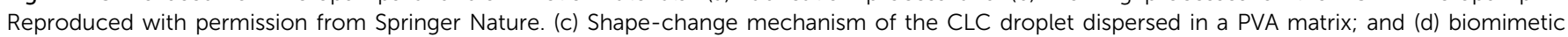
octopus derived from the CLC droplet dispersed in the PVA matrix. ${ }^{157}$ Reproduced with permission from Wiley. 
switching, such as RGB LED backlights. ${ }^{158}$ In order to expand the $\mathrm{BP}$ temperature range while realizing a low working voltage, BPLC-Ms consisting of inner BPLC cores and outer PU shells have been proposed recently. ${ }^{40}$ The thermal stability of the BPLCs is greatly enhanced by the surface anchoring and physical confinement brought by the PU shell, leading to a BP temperature range of about $15{ }^{\circ} \mathrm{C}$. Besides, a brand new electro-optical response mechanism which only drives the surface BPLCs is exploited to reduce the working voltage. When the electric field is applied to the BPLC-Ms, the inner BPLCs retain the double helix structure, while the BPLCs that are near the shell interface reorient into a homeotropic direction, resulting in a hierarchical BP structure and a dark green color. As a result, an ultra-low working electric field of less than $0.55 \mathrm{~V} \mathrm{~mm}^{-1}$ is realized, far lower than that of the reported BPLCs. Such microcapsules provide a multi-dimensional strategy for further development of stimuli-responsive materials.

\section{Conclusion and outlook}

The LCs show fascinating stimuli-responsive properties and optical properties, with applications over a wide range of areas. Nevertheless, the stability and versatility of the LCs are severely limited by their fluidity. Although modified LCs with a homogeneous polymer matrix can solve the problem to some extent, the utilization of the polymer matrix also reduces the responsive sensitivity. The phase separation of the polymer matrix and the LCs has triggered the development of LC-Ms, which consist of an inner LC core and an outer polymer shell, overcoming the limitations and providing a useful platform for numerous applications. The fabrication of LC-Ms is achieved via LC emulsification and shell formation. Some bulk emulsification strategies, such as stirring, membrane emulsification and ultrasonication, have enabled high throughput while showing poor controllability. By contrast, microfluidics can ensure monodisperse LC droplets, while the production throughput remains low. The as-prepared LC-Ms exhibit stimuli-responsive configuration transition properties for which the molecular orientation can change under external stimuli such as an electric field, light, and temperature, leading to a variation of the optical properties. For the CLC-Ms, the unique photonic stopband and photonic communication are difficult to implement with other materials. These performance parameters can be tuned by either regulating the chemical composition or changing the microstructure. Based on these attractive characteristics, the applications of LC-Ms in the field of sensing, anti-counterfeiting and smart fabrics have been investigated in depth.

In spite of the rising research enthusiasm for the LC-Ms, there remain some non-negligible problems. For example, the bulk fabrication of LC-Ms with a high size uniformity, stability, and optical performance has not yet been realized. Further improvements in microfluidics are of urgent need to enable the scaled-up production of monodisperse LC-Ms. The interaction principle between the core and the shell, as well as between LC molecules, needs to be studied at the molecular scale, using either theoretical simulations or systematic experiments to better guide the design and construction of LC-Ms. Moreover, the combination of existing LC techniques and newborn LC-Ms should be further studied, which on the one hand can improve existing LC techniques, and on the other hand expand the application field for LC-Ms.

For the investigation of the LC-M smart textiles, current studies are being devoted to the achievement of electrochromic and thermochromic properties. Further research and development is needed for the textiles to become more versatile. Specific receptors that can detect harmful molecules in the atmosphere could be bound on the polymer shells to endow the textiles with the function of individual protection. The durability of textiles should be improved for their long-term use by either introducing covalent bonds into the textiles or modifying the surface with superhydrophobic coatings, which can endow the textiles with self-cleaning and abrasive-resistance attributes. Furthermore, the luminosity and color purity decreased distinctly after the LCs were processed into textiles, which is imperative for improvement. Optimizing processing conditions and developing new skeletal materials would be alternative solutions.

Another key requirement is to achieve LC-M sensors with gas-detection properties. The existing LC gas sensors suffer from low sensitivity. For example, the state-of-the-art LC chlorine sensors have realized a detection limit of about $200 \mathrm{ppm}$, which is far beyond the standard formulated by the American Conference of Government Industrial Hygienist (0.5 ppm at work sites). ${ }^{159}$ This is because the intrinsic structure of the traditional LC sensors is unsuitable for gas detection, i.e., the gas-combining receptors, which are modified on the glass substrate, are inevitably covered by the LCs, and thus the combination of the analyte and the receptor is severely impeded. Encapsulating the LCs into the microcapsules can on the one hand expose the receptor to the external environment, and on the other hand increase the specific surface area of the sensor, thus solving the problem. At the present stage, there is an urgent need to explore the strategy of binding the gascombining receptors on the polymer shells to promote the realization of efficient LC-M gas sensors.

Besides, considering that the LC-Ms show great potential in cell-free applications, they are supposed to realize the real-time monitoring of the physiological signals either in the human body or on the body's surface. As a result, the biocompatibility of the LC-Ms needs to be investigated. The utilization of nontoxic and inert ingredients such as silica gel, hydrogel and titanium would be a promising approach for improving the biocompatibility. The long-term stability of the LC-Ms, which can prevent the leakage of the LC molecules, is also important for clinical applications, especially for applications in the body. Therefore, the investigation of a new shell type, which can provide the LC-Ms with both stability and biocompatibility, as well as maintaining outstanding transparency, is an important task.

The demand for developing a precise and systematic procedure for optical pattern classification and performance prediction should also be emphasized. These tasks are time 
consuming and not humanly feasible. Specifically, for the LC-M sensors, a tiny difference in the optical patterns usually implies a distinction of the analyte type and concentration. For anticounterfeiting LC-M devices, a similar but counterfeit sample should be distinguished from a large amount of samples efficiently. During fabrication of the LC-Ms, the responsive and optical performance could be predicted based on the molecular structures of the ingredients. Machine learningbased strategies can eliminate the human factor and seem to be an ideal solution. Overall, current studies are strongly embodying the superiority of LC-Ms in a variety of applications. The devices derived from LC-Ms can be easy to use and assemble, as well as be incorporated in other rigid or flexible materials. As a result, LC-Ms show great research value and promising application potential, especially after the remaining problems have been solved.

\section{Abbreviations}

$\begin{array}{ll}\text { LC } & \text { Liquid crystal } \\ \text { NLC } & \text { Nematic liquid crystal } \\ \text { CLC } & \text { Chiral nematic liquid crystal } \\ \text { LC-Ms } & \text { Liquid crystal microcapsules } \\ \text { LCP } & \text { Liquid crystal polymer } \\ \text { LCE } & \text { Liquid crystal elastomer } \\ \text { LCN } & \text { Liquid crystal network } \\ \text { DDLC } & \text { Dye-doped liquid crystal } \\ \text { MDLC } & \text { Metal-doped liquid crystal } \\ \text { SE } & \text { Surface energy } \\ \text { PVA } & \text { Polyvinyl alcohol } \\ \text { PMMA } & \text { Polymethyl methacrylate } \\ \text { PU } & \text { Polyurethane } \\ \text { SDS } & \text { Sodium dodecyl sulfate } \\ \text { CTAB } & \text { Hexadecyl trimethyl ammonium bromide } \\ \text { DTAB } & \text { Dodecyl trimethyl ammonium bromide } \\ \text { SIPS } & \text { Solvent-evaporation induced phase separation } \\ \text { PEGDA } & \text { Poly(ethylene glycol) diacrylate } \\ \text { MDI } & \text { Diphenyl-methane-diisocyanate } \\ \text { TDI } & \text { Toluene diisocyanate } \\ \text { IPDI } & \text { Isophorone diisocyanate } \\ \text { PI } & \text { Polyimide } \\ \text { HTP } & \text { Helical twisting power } \\ \text { PBG } & \text { Photonic bandgap } \\ \text { IgG } & \text { Immunoglobulin G } \\ \text { TEPA } & \text { Tetraethylenepentamine } \\ \text { PAA } & \text { Poly(acrylic acid) } \\ \text { LCP } & \text { Poly(4-cyanobiphenyl-4'-oxyundecyl acrylate) } \\ \text { EDC } & \text { 1-(3-Dimethylaminopropyl)-3-ethylcarbodiimide } \\ \text { NHS } & \text { N-Hydroxysuccinimide } \\ \text { DMMP } & \text { Dimethyl methylphosphonate } \\ \text { R-CPL } & \text { Right-circularly polarized light } \\ \text { L-CPL } & \text { Left-circularly polarized light } \\ \text { FCLC } & \text { Fluorescent chiral nematic liquid crystal } \\ \text { RMs } & \text { Reactive mesogens } \\ \text { RECs } & \text { Rare-earth complexes } \\ & \end{array}$
PUF
PVP
Physical unclonable function
PEO
VTES Vinyl-trimethyl-silane
EDGMA Ethylene glycol dimethacrylate
TFEMA Trifluoroethyl methacrylate
COC Cholesteryl oleyl carbonate
CPE Cholesterol pelargonate
BPLC Blue phase liquid crystal

\section{Conflicts of interest}

There are no conflicts to declare.

\section{Acknowledgements}

We acknowledge funding from the Research Initiation Project 113010-PI2001 and Major Research Project of Zhejiang Laboratory.

\section{References}

1 H. K. Bisoyi and S. Kumar, Chem. Soc. Rev., 2011, 40, 306-319.

2 B. R. Kaafarani, Chem. Mater., 2011, 23, 378-396.

3 J. P. F. Lagerwall and G. Scalia, Curr. Appl. Phys., 2012, 12, 1387-1412.

4 C. Boyon, V. Soldan and M. Mitov, ACS Appl. Mater. Interfaces, 2021, 13, 30118-30126.

5 A. Jákli, Liq. Cryst. Rev, 2013, 1, 65-82.

6 M. Mitov, ChemPhysChem, 2014, 15, 1245-1250.

7 F. Reinitzer, Monatsh. Chem., 1888, 9, 421-441.

8 M. Draper, I. M. Saez, S. J. Cowling, P. Gai, B. Heinrich, B. Donnio, D. Guillon and J. W. Goodby, Adv. Funct. Mater., 2011, 21, 1260-1278.

9 R. S. Kularatne, H. Kim, J. M. Boothby and T. H. Ware, J. Polym. Sci. Pol. Phys, 2017, 55, 395-411.

10 M. Liu, L. Zhang and T. Wang, Chem. Rev., 2015, 115, 7304-7397.

11 C. Tschierske, Angew. Chem., Int. Ed., 2013, 52, 8828-8878.

12 Z. Xu and C. Gao, ACS Nano, 2011, 5, 2908-2915.

13 P. S. Simonario, T. M. de Andrade and F. C. M. Freire, Braz. J. Phys., 2016, 46, 26-34.

14 D. K. Sahu, T. G. Anjali, M. G. Basavaraj, J. Aplinc, S. Copar and S. Dhara, Sci. Rep., 2019, 9, 81.

15 K. Kristiansen, H. Zeng, B. Zappone and J. N. Israelachvili, Langmuir, 2015, 31, 3965-3972.

16 J. J. Schwartz, A. M. Mendoza, N. Wattanatorn, Y. Zhao, V. T. Nguyen, A. M. Spokoyny, C. A. Mirkin, T. Bas and P. S. Weiss, JACS, 2016, 138, 5957-5967.

17 P. Guillamat, J. Ignes-Mullol and F. Sagues, Proc. Natl. Acad. Sci. U. S. A., 2016, 113, 5498-5502.

18 A. A. Shah, H. Kang, K. L. Kohlstedt, K. H. Ahn, S. C. Glotzer, C. W. Monroe and M. J. Solomon, Small, 2012, 8, 1551-1562.

19 R. Basu, Phys. Rev. E, 2021, 103, 022710. 
20 Y. Chen and S. Wu, Appl. Phys. Lett., 2013, 102, 171110.

21 Y. Kim, M. Lee, H. S. Wang and K. Song, Liq. Cryst., 2018, 45, 757-764.

22 W. Wei, Y. Xia, S. Ettinger, S. Yang and A. G. Yodh, Nature, 2019, 576, 433-436.

23 J. Chen, C. Huang and C. Chao, ACS Appl. Mater. Interfaces, 2014, 6, 6757-6764.

24 M. Mathews and N. Tamaoki, JACS, 2008, 130, 11409-11416.

25 R. Dabrowski, P. Kula and J. Herman, Crystals, 2013, 3, 443-482.

26 J. Xie, T. Zeng, Y. Zhou, Y. Yuan, Y. Zhou, T. Lin, X. Jiang, F. Fan and S. Wen, Liq. Cryst., 2021, DOI: 10.1080/ 02678292.2021.1909766.

27 P. Gan, X. Zhang, L. Zhao, W. Shi, H. Cao, H. Wang, Z. Yang, D. Wang and W. He, Liq. Cryst., 2021, DOI: 10.1080/02678292.2021.1909765.

28 M. W. Geis, P. J. Bos, V. Liberman and M. Rothschild, Opt. Express, 2016, 24, 13812-13823.

29 J. Yan, L. Rao, M. Jiao, Y. Li, H. Cheng and S. Wu, J. Mater. Chem., 2011, 21, 7870-7877.

30 H. Lu, S. Wu, C. Zhang, L. Qiu, X. Wang, G. Zhang, J. Hu and J. Yang, Dyes Pigm., 2016, 128, 289-295.

31 M. A. El-Rabiaey and S. S. A. Obayya, J. Lightwave Technol., 2016, 34, 3726-3732.

32 D. J. Mulder, A. P. H. J. Schenning and C. W. M. Bastiaansen, J. Mater. Chem. C, 2014, 2, 6695-6705.

33 H. Zeng, O. M. Wani, P. Wasylczyk, R. Kaczmarek and A. Priimagi, Adv. Mater., 2017, 29, 1701814.

34 T. J. White and T. J. White, Nat. Mater., 2015, 14, 1087-1098.

35 J. Lv, Y. Liu, J. Wei, E. Chen, L. Qin and Y. Yu, Nature, 2016, 537, 179-184.

36 L. Haan, C. Sunchez-Somolinos, C. M. W. Bastiaansen, A. Schenning and D. J. Broer, Angew. Chem., Int. Ed., 2012, 51, 12469-12472.

37 D. J. Broer, C. M. W. Bastiaansen, M. G. Debije and A. P. H. J. Schenning, Angew. Chem., Int. Ed., 2012, 51, 7102-7109.

38 H. Jiang, Y. Tang, X. Zeng, R. Xiao, P. Lv, L. Wang and Y. Lu, Chin. Opt. Lett., 2020, 18, 031201.

39 H. Zhang, S. Tian, Q. Wang, D. Wang and T. Zhao, Liq. Cryst., 2021, 48, 1065-1072.

40 P. Lin, Z. Wei, Q. Yan, Y. Chen, M. Wu, J. Xie, M. Zeng, W. Wang, J. Xu and Z. Cheng, J. Mater. Chem. C, 2019, 7, 4822-4827.

41 S. S. Lee and S. Kim, Macromol. Res., 2018, 26, 1054-1065.

42 M. Kim, K. J. Park, S. Seok, J. M. Ok, H. Jung, J. Choe and D. H. Kim, ACS Appl. Mater. Interfaces, 2015, 7, 17904-17909.

43 J. Wang, A. Jakli, Y. Guan, S. Fu and J. West, J. Soc. Inf. Display, 2017, 33, 16-20.

44 M. Humar, Liq. Cryst., 2016, 43, 1937-1950.

45 Y. Maa and C. Hsu, J. Controlled Release, 1996, 38, 219-228.

46 J. M. Shin, M. P. Kim, H. Yang, K. H. Ku, S. G. Jang, K. H. Youm, G. Yi and B. J. Kim, Chem. Mater., 2015, 27, 6314-6321.

47 S. G. Gaikwad and A. B. Pandit, Ultrason. Sonochem., 2008, 15, 554-563.
48 S. Sumitomo, M. Ueta, M. A. Uddin and Y. Kato, Chem. Eng. Technol., 2019, 42, 381-387.

49 J. P. Lorimer and T. J. Mason, Chem. Soc. Rev., 1987, 16, 239-247.

50 M. K. Li and H. S. Fogler, J. Fluid Mech., 1978, 88, 499-511.

51 D. M. Higgins and D. M. Skauen, J. Pharm. Sci., 1972, 61, 1567-1570.

52 S. M. Joscelyne and G. Tragardh, J. Membr. Sci., 2000, 169, 107-117.

53 W. Yu, B. Li, X. Liu and Y. Chen, Chem. Eng. Sci., 2021, 243, 116648.

54 M. B. Romanowsky, A. R. Abate, A. Rotem, C. Holtzec and D. A. Weitz, Lab Chip, 2012, 12, 802-807.

55 W. Wang, M. Zhang and L. Chu, Acc. Chem. Res., 2014, 47, 373-384.

56 L. T. Skeggs, Am. J. Clin. Pathol., 1957, 27, 311-322.

57 G. Whitesides and A. Stroock, Phys. Today, 2001, 54, 42-48.

58 J. C. McDonald, D. C. Duffy, J. R. Anderson, D. T. Chiu, H. Wu, O. J. A. Schueller and G. M. Whitesides, Electrophoresis, 2000, 21, 27-40.

59 M. Costa, I. Pinho, M. V. Loureiro, A. C. Marques, C. L. Simoes and R. Simoes, Polym. Bull., 2021, DOI: 10.1007/s00289-021-03690-1.

60 A. S. Utada, L. Y. Chu, A. Fernandez-Nieves, D. R. Link, C. Holtze and D. A. Weitz, MRS Bull., 2007, 32, 702-708.

61 R. K. Shah, H. C. Shum, A. C. Rowat, D. Lee, J. J. Agresti, A. S. Utada, L. Chu, J. K. Alberto, F. Nieves, C. J. Martinez and D. A. Weitz, Mater. Today, 2008, 11, 18-27.

62 S. S. Lee, S. K. Kim, J. C. Won, Y. H. Kim and S. Kim, Angew. Chem., Int. Ed., 2015, 54, 15266-15270.

63 S. S. Lee, H. J. Seo, Y. H. Kim and S. Kim, Adv. Mater., 2017, 29, 1606894.

64 U. Ali, J. B. Bt, A. Karim and N. A. Buang, Polym. Rev., 2015, 55, 678-705.

65 J. Xu, Y. Tsai and S. Hsu, Molecules, 2020, 25, 5296.

66 E. M. Ahmed, J. Adv. Res., 2015, 6, 105-121.

67 E. Delebecq, J. Pascault, B. Boutevin and F. Ganachaud, Chem. Rev., 2013, 113, 80-118.

68 S. Kulkarni and P. Thareja, Surf. Rev. Lett., 2016, 24, 1750044.

69 Y. Jung, M. Khan and S. Park, J. Mater. Chem. B, 2014, 2, 4922-4928.

70 K. Lv, D. Liu, W. Li, S. Tang and X. Zhou, Mol. Cryst. Liq. Cryst., 2012, 557, 217-227.

71 S. S. Lee, B. Kim, S. K. Kim, J. C. Won, Y. H. Kim and S. Kim, Adv. Mater., 2015, 27, 627-633.

72 Y. Guan, L. Zhang, D. Wang, J. L. West and S. Fu, Mater. Des., 2018, 147, 28-34.

73 C. Chen, I. Tseng, H. Lu, W. Tseng, M. Tsai and S. Huang, Mater. Sci. Eng., A, 2011, 528, 4917-4923.

74 Q. Wang, H. Xu, S. Zhou, S. Garnier, S. Fuldner, L. Ye and Z. Feng, J. Coat. Technol. Res., 2017, 14, 1279-1288.

75 F. Alizadegana, S. M. Mirabedinia, S. Pazokifarda, S. G. Moghadama and R. Farnood, Prog. Org. Coat., 2018, 123, 350-361.

76 W. J. Lee, B. Kim, S. W. Han, M. Seo, S. Choi, H. Yang, S. Kim, S. Jeong and J. W. Kim, J. Ind. Eng. Chem., 2018, 68, 393-398. 
77 C. Esteves, E. Ramou, A. R. P. Porteira, A. J. M. Barbosa and A. C. A. Roque, Adv. Opt. Mater., 2020, 8, 1902117.

78 A. A. Sharma and J. P. F. Lagerwall, Liq. Cryst., 2018, 45, 2319-2328.

79 B. S. Murray, R. A. Pelcovits and C. Rosenblatt, Phys. Rev. E: Stat., Nonlinear, Soft Matter Phys., 2014, 90, 052501.

80 J. Engel, M. J. Ramsey-Musolf and U. Kolck, Prog. Part. Nucl. Phys., 2013, 71, 21-74.

81 H. Sun, Z. Xie, C. Ju, X. Hu, D. Yuan, W. Zhao, L. Shui and G. Zhou, Polymers, 2019, 11, 694.

82 H. H. Khalighab, K. Liewc, Y. Han, N. M. Abukhdeir and I. Goldthorpe, Sol. Energy Mater. Sol. C, 2015, 132, 337-341.

83 H. K. Bisoyi, T. J. Bunning and Q. Li, Adv. Mater., 2018, 30, 1706512.

84 D. Wang and X. Wang, Prog. Polym. Sci., 2013, 38, 271-301.

85 D. Martella, S. Nocentini, F. Micheletti, D. S. Wiersma and C. Parmeggiani, Soft Matter, 2019, 15, 1312-1318.

86 N. Park, S. Park and K. Suh, Liq. Cryst., 2002, 29, 1253-1258. 87 J. Lin, M. Hsieh, G. Wei, T. Mo, S. Huang and C. Lee, Opt. Express, 2013, 21, 15765-15776.

88 P. V. Shibaev, O. Roslyak, E. Gullatt, J. Plumitallo and U. Aparajita, Appl. Phys. A: Mater., 2020, 126, 920.

89 L. Dai, W. Li, J. Cao, J. Li and S. Liu, Prog. Chem., 2015, 27, 861-869.

90 M. Mitov, Adv. Mater., 2012, 24, 6260-6276.

91 M. Jeong and K. Kwak, Appl. Opt., 2016, 55, 9378-9383.

92 M. Jakovljevic, B. Lozo and M. K. Gunde, Color. Technol., 2017, 133, 81-87.

93 M. Mathews, R. S. Zola, S. Hurley, D. Yang, T. J. White, T. J. Bunning and Q. Li, JACS, 2010, 132, 18361-18366.

94 H. Chen, X. Wang, H. Bisoyi, L. Chen and Q. Li, Langmuir, 2021, 37, 3789-3807.

95 K. Lv, D. Liu, W. Li, Q. Tian and X. Zhou, Dyes Pigm., 2012, 94, 452-458.

96 P. Kumar, V. Sharma, C. Jaggi and K. K. Raina, Liq. Cryst., 2017, 44, 757-767.

97 J. Erdmann, J. W. Doane, S. Zumer and G. Chidichimo, Proc. SPIE, 1989, 1080, 32-44.

98 M. Sheng, L. Zhang, Y. Guan, L. Li and S. Fu, J. Mol. Liq., 2019, 283, 816-822.

99 P. Kumar, V. Sharma, C. Jaggi, P. Malik and K. K. Raina, Liq. Cryst., 2017, 44, 757-767.

100 K. G. Mishra, S. K. Dubey, S. A. Mani and M. S. Pradhan, J. Mol. Liq., 2016, 224, 668-671.

101 H. Peng, W. Jiang, Q. Liu, G. Chen, M. Ni, F. Liang, Y. Liao, X. Xie and I. I. Smalyukh, Langmuir, 2018, 34, 10955-10963.

102 M. Sheng, L. Zhang, Q. Lei, A. Hu, L. Li and S. Fu, Dyes Pigm., 2020, 180, 108544.

103 I. Lee, J. Noh, J. Lee and T. Kim, ACS Appl. Mater. Interfaces, 2017, 9(42), 37395-37401.

104 S. J. Woltman, G. D. Jay and G. P. Crawford, Nat. Mater., 2017, 6, 929-938.

105 M. Khan, W. Li, S. Mao, S. Shah and J. Lin, Adv. Sci., 2019, 6, 1900778.

106 S. Sun, M. Lee, Y. Lee, W. Lee, X. Song and C. Chen., Biomed. Opt. Express, 2015, 6, 245-256.
107 L. Zhu, J. Wang, J. Liu, L. Wang and W. Yan, Prog. Chem., 2020, 32, 344-360.

108 V. M. Aroutiounian, J. Contemp. Phys. Arme, 2019, 54, 356-367.

109 N. Popov, L. W. Honaker, M. Popova, N. Usol'tseva, E. K. Mann, A. Jákli and P. Popov, Materials, 2018, 11, 20.

110 K. Lee, K. C. Gupta, S. Park and I. Kang, J. Mater. Chem. B, 2016, 4, 704-715.

111 G. Han and C. Jang, Talanta, 2014, 128, 44-50.

112 P. Hong, K. Yun and C. Jang, BioChip J., 2021, 15, 152-161.

113 T. D. S. Duong and C. Jang, Colloids Surf., A, 2020, 604, 125304.

114 X. Han, D. Han, J. Zeng, J. Deng, N. Hu and J. Yang, Microchem. J., 2020, 157, 105057.

115 J. M. Dietschy, Arch. Intern. Med., 1972, 130, 473-474.

116 L. Zhou, Q. Hu, Q. Kang and L. Yu, Talanta, 2018, 190, 375-381.

117 P. Lin, Q. Yan, Z. Wei, Y. Chen, S. Chen, H. Wang, Z. Huang, X. Wang and Z. Cheng, ACS Appl. Mater. Interfaces, 2018, 10, 18289-18299.

118 Y. Iwai, H. Kaji, Y. Uchida and N. Nishiyam, J. Mater. Chem. C, 2014, 2, 4904-4908.

119 M. Yang, J. Huang, J. Fan, J. Du, K. Pu and X. Peng, Chem. Soc. Rev., 2020, 49, 6800-6815.

120 H. Lee, S. Munir and S. Park, ACS Appl. Mater. Interfaces, 2016, 8, 26407-26417.

121 A. Kucühler, M. Yoshimoto, S. Luginbuhl, F. Mavelli and P. Walde, Nat. Nanotechnol., 2016, 11, 409-420.

122 Z. He, G. Li, J. Chen, Y. Huang, T. An and C. Zhang, Environ. Int., 2015, 77, 85-94.

123 X. Niu, Y. Zhong, R. Chen, F. Wang and D. Luo, Opt. Express, 2017, 25, 13549-13556.

124 S. S. Sridharamurthy, K. D. Cadwell, N. L. Abbott and H. Jiang, IEEE Sens., 2007, 1044-1047.

125 S. Nordlund and M. Hogbom, FEBS J., 2013, 280, 3484-3490.

126 H. Dinh, E. Nakata, P. Lin, M. Saimura, H. Ashida and T. Morii, Bioorg. Med. Chem., 2019, 27, 115120.

127 S. Sayed, L. Pascual, M. Licchelli, R. Martinez-Manez, S. Gil, A. M. Costero and F. Sancenon, ACS Appl. Mater. Interfaces, 2016, 8, 14318-14322.

128 C. Yang, L. Gu, C. Ma, M. Gu, X. Xie, H. Shi, H. Ma, W. Yao, Z. An and W. Huang, Mater. Horiz., 2019, 6, 984-989.

129 B. Song, H. Wang, Y. Zhong, B. Chu, Y. Su and Y. He, Nanoscale, 2018, 10, 1617-1621.

130 J. Guo, J. Zhang, Q. Zhang, N. Jiang and J. Wei, RSC Adv., 2013, 3, 21620-21627.

131 H. J. Seo, S. S. Lee, J. Noh, J. Ka, J. C. Won, C. Park, S. Kim and Y. H. Kim, J. Mater. Chem. C, 2017, 5, 7567-7573.

132 L. Qin, X. Liu, K. He, G. Yu, H. Yuan, M. Xu, F. Li and Y. Yu, Nat. Commun., 2021, 12, 699.

133 P. Lin, H. Chen, A. Li, H. Zhuang, Z. Chen, Y. Xie, H. Zhou, S. Mo, Y. Chen, X. Lu and Z. Cheng, ACS Appl. Mater. Interfaces, 2020, 12, 46788-46796.

134 J. Noh, H. Liang, I. Drevensek-Olenik and J. P. F. Lagerwall, J. Mater. Chem. C, 2014, 2, 806-810. 
135 Y. Geng, J. Noh, I. Drevensek-Olenik, R. Rupp, G. Lenzini and J. P. F. Lagerwall, Sci. Rep., 2016, 6, 26840.

136 M. Amjadi, K. Kyung, I. Park and M. Sitti, Stretchable, Adv. Funct. Mater., 2016, 26, 1678-1698.

137 W. Zeng, L. Shu, Q. Li, S. Chen, F. Wang and X. Tao, Adv. Mater., 2014, 26, 5310-5336.

138 M. Stoppa and A. Chiolerio, Sensors, 2014, 14, 11957-11992.

139 O. Wiese, D. Marenduzzo and O. Henrich, Soft Matter, 2016, 12, 9223-9237.

140 D. V. Shmeliova, S. V. Pasechnik, S. S. Kharlamov, A. V. Zakharov, E. P. Pozhidaev, V. A. Barbashov and T. P. Tkachenko, Crystals, 2020, 10, 1029.

141 N. Bhardwaj and S. C. Kundu, Biotechnol. Adv., 2010, 28, 325-347.

142 S. Arumugam, Y. Li, S. Senthilarasu, R. Torah, A. L. Kanibolotsky, A. R. Inigo, P. J. Skabarac and S. P. Beebya, J. Mater. Chem. A, 2016, 4, 5561-5568.

143 H. Tseng, L. Chang, K. Lin, C. Li, W. Lin, C. Wang, C. Lin, S. Liu and T. Lin, Materials, 2020, 13, 4137.

144 M. Sheng, L. Zhang, D. Wang, M. Li, L. Li, J. L. West and S. Fu, Dyes Pigm., 2018, 158, 1-11.

145 M. Sheng, L. Zhang, S. Jiang, L. Yang, F. Zaaboul and S. Fu, ACS Appl. Mater. Interfaces, 2021, 13(11), 13586-13595.

146 M. Sheng, L. Zhang, J. L. West and S. Fu, ACS Appl. Mater. Interfaces, 2020, 12, 29728-29736.

147 M. Sheng, J. Li, X. Jiang, C. Wang, J. Li, L. Zhang and S. Fu, ACS Appl. Mater. Interfaces, 2021, 13, 33282-33290.
148 L. W. Honaker, S. Vats, M. Anyfantakis and J. P. F. Lagerwall, J. Mater. Chem. C, 2019, 7, 11588-11596.

149 X. Niu, J. Liu, W. Liu and S. Zhu, Liq. Cryst., 2019, 46, 1396-1402.

150 O. T. Picot, M. Dai, D. J. Broer, T. Peijs and C. W. M. Bastiaansen, ACS Appl. Mater. Interfaces, 2013, 5, 7117-7121.

151 H. Hao and X. Liu, Fibers Polym., 2017, 18, 246-252.

152 Y. Guan, D. M. Agra-Kooijman, S. Fu, A. Jakli and J. L. West, Adv. Mater., 2019, 31, 1902168.

153 J. Wang, J. Kolacz, Y. Chen, A. Jakli, J. Kawalec, M. Benitez and J. L. West, SID Int. Symp. Dig. Tech. Pap., 2017, 48, 147-149.

154 E. Fleischmann, H. Liang, N. Kepernaum, F. Giesselmann, J. Lagerwall and R. Zentel, Nat. Mater., 2012, 3, 1178.

155 C. M. Spillmann, J. Naciri, W. R. Algar, I. L. Medintz and J. B. Delehanty, ACS Nano, 2014, 8, 6986-6997.

156 Y. Iwai, T. Maeda, Y. Uchida, F. Araoka and N. Nishiyama, Adv. Photonics Res., 2021, 2, 2000079.

157 C. Yang, B. Wu, J. Ruan, P. Zhao, L. Chen, D. Chen and F. Ye, Adv. Mater., 2021, 33, 2006361.

158 K. Chen, S. Gauza, H. Xianyu and S. Wu, J. Disp. Technol., 2010, 6, 49-51.

159 T. Szilvasi, N. Bao, K. Nayani, H. Yu, P. Rai, R. J. Twieg, M. Mavrikakis and N. L. Abbott, Angew. Chem., Int. Ed., 2018, 57, 9665-9669. 